\title{
Effects of Epoxyeicosatrienoic Acid-Enhancing Therapy on the Course of Congestive Heart Failure in Angiotensin II-Dependent Rat Hypertension: From mRNA Analysis towards Functional In Vivo Evaluation
}

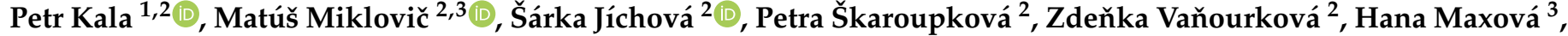 \\ Olga Gawrys ${ }^{2,4}{ }^{\oplus}$, Elzbieta Kompanowska-Jezierska ${ }^{4}$, Janusz Sadowski ${ }^{4}{ }^{\oplus}$, John D. Imig ${ }^{5}$, John R. Falck ${ }^{6}{ }^{\oplus}$, \\ Josef Veselka ${ }^{1}$, Luděk Červenka ${ }^{2,3}$, Renáta Aiglová ${ }^{7}$, Marek Vícha ${ }^{7}$, Vít Gloger ${ }^{7}$ and Miloš Táborský ${ }^{7, *}$
}

\section{check for} updates

Citation: Kala, P.; Miklovič, M.; Jíchová, Š.; Škaroupková, P.; Vaňourková, Z.; Maxová, H.; Gawrys, O.; Kompanowska-Jezierska, E.; Sadowski, J.; Imig, J.D.; et al. Effects of Epoxyeicosatrienoic Acid-Enhancing Therapy on the Course of Congestive Heart Failure in Angiotensin II-Dependent Rat Hypertension: From mRNA Analysis towards Functional In Vivo Evaluation. Biomedicines 2021, 9, 1053. https://doi.org/10.3390/ biomedicines 9081053

Academic Editors: Iveta Bernatova, Monika Bartekova and Silvia Liskova

Received: 8 July 2021

Accepted: 18 August 2021

Published: 20 August 2021

Publisher's Note: MDPI stays neutral with regard to jurisdictional claims in published maps and institutional affiliations.

Copyright: (c) 2021 by the authors. Licensee MDPI, Basel, Switzerland. This article is an open access article distributed under the terms and conditions of the Creative Commons Attribution (CC BY) license (https:// creativecommons.org/licenses/by/ $4.0 /)$.
1 Department of Cardiology, University Hospital Motol and 2nd Faculty of Medicine, Charles University, 15006 Prague, Czech Republic; petrkala@gmail.com (P.K.); josef.veselka@fnmotol.cz (J.V.)

2 Center for Experimental Medicine, Institute for Clinical and Experimental Medicine, 14021 Prague, Czech Republic; mixm@ikem.cz (M.M.); savr@ikem.cz (Š.J.); pesp@ikem.cz (P.Š.); zdva@ikem.cz (Z.V.); olga.gawrys@ikem.cz (O.G.); luce@ikem.cz (L.Č.)

3 Department of Pathophysiology, 2nd Faculty of Medicine, Charles University, 15006 Prague, Czech Republic; hana.maxova@lfmotol.cuni.cz

4 Department of Renal and Body Fluid Physiology, Mossakowski Medical Research Institute, Polish Academy of Sciences, 02-106 Warsaw, Poland; ekompanowska@imdik.pan.pl (E.K.-J.); jsadowski@imdik.pan.pl (J.S.)

5 Drug Discovery Center, Medical College of Wisconsin, Wauwatosa, WI 53226, USA; jdimig@mcw.edu

6 Department of Biochemistry, University of Texas Southwestern Medical Center, Dallas, TX 75390, USA; j.falck@utsouthwestern.edu

7 Department of Internal Medicine I, Cardiology, University Hospital Olomouc and Palacký University, I.P. Pavlova 185/6, Nová Ulice, 77900 Olomouc, Czech Republic; dr.renata.aiglova@seznam.cz (R.A.); dr.marek.vicha@seznam.cz (M.V.); dr.vit.gloger@seznam.cz (V.G.)

* Correspondence: prof.Milos.Taborsky@seznam.cz

Abstract: This study evaluates the effects of chronic treatment with EET-A, an orally active epoxyeicosatrienoic acid (EETs) analog, on the course of aorto-caval fistula (ACF)-induced heart failure (HF) in Ren-2 transgenic rats (TGR), a model characterized by hypertension and augmented activity of the renin-angiotensin system (RAS). The results were compared with standard pharmacological blockade of the RAS using angiotensin-converting enzyme inhibitor (ACEi). The rationale for employing EET-A as a new treatment approach is based on our findings that apart from increased RAS activity, untreated ACF TGR also shows kidney and left ventricle (LV) tissue deficiency of EETs. Untreated ACF TGR began to die 17 days after creating ACF and were all dead by day 84 . The treatment with EET-A alone or ACEi alone improved the survival rate: in 156 days after ACF creation, it was $45.5 \%$ and $59.4 \%$, respectively. The combined treatment with EET-A and ACEi appeared to improve the final survival to $71 \%$; however, the difference from either single treatment regimen did not reach significance. Nevertheless, our findings support the notion that targeting the cytochrome P-450-dependent epoxygenase pathway of arachidonic acid metabolism should be considered for the treatment of HF.

Keywords: congestive heart failure; volume-overload heart failure; aorto-caval fistula; hypertension; Ren-2 transgenic rats; cytochrome P-450; epoxyeicosatrienoic acids; renin-angiotensin system; angiotensin-converting enzyme inhibitor

\section{Introduction}

Heart failure (HF) has become a major public health problem, affecting currently more than 6.5 and 9.2 million people in the United States of America and the European Union, respectively; the yearly increase in the number of new HF patients is estimated at more 
than 1.1 million [1,2]. Despite an array of therapeutic approaches available and recent pharmacological advances, the prognosis in HF is still poor, in fact, worse than in common cancers [1,3-6]. Evidently, new treatment strategies are urgently needed as well as focused experimental studies to evaluate the therapeutic effects of new therapeutic approaches.

Recent research has been focused on the epoxyeicosatrienoic acids (EETs), the metabolites of cytochrome P-450 (CYP)-dependent epoxygenase pathway of arachidonic acid (AA) metabolism. It was shown that EETs importantly contribute to the regulation of renal and cardiovascular function and exert antihypertensive and organ-protective actions [7-10]. It was also proposed that intrarenal EETs operate as an endogenous compensatory system opposing increased renin-angiotensin system (RAS) activity [7-9,11]. Hypertension and inappropriately activated RAS are essential factors promoting the progression of HF $[1,2,12-16]$; hence, the therapeutic potential of EETs in HF seems promising. EETs are rapidly transformed by soluble epoxide hydrolase $(\mathrm{sEH})$ to biologically inactive dihydroxyeicosatrienoic acids (DHETEs) [7-9,17]. Therefore, in most earlier studies, sEH inhibition was employed, and antihypertensive, cardio-, and renoprotective effects were reported [7-9]. However, this strategy might prove less successful whenever endogenous EETs biosynthesis is compromised, which might be the case in HF patients receiving the drugs that inhibit CYP activity as an unsolicited action [17-19]. Therefore, an alternative approach, which circumvents this limitation, consists of applying EETs-agonistic analogs designed to resist degradation. This new approach has not yet been adequately explored: the obtained results were not entirely consistent and not yet comprehensively evaluated in HF [20-25].

In this study, we used Ren-2 transgenic rats (TGR) with HF induced by aorto-caval fistula (ACF). ACF presents a well-defined model of heart failure due to volume overload, characterized by activation of the RAS, congestion, and impairment of renal function. The model has many features in common with untreated human HF [26-32] and is recommended by the American Heart Association and the European Society of Cardiology for preclinical testing to identify new targets for the treatment of HF patients [33,34]. The TGR model combines activation of the RAS and hypertension [35,36], two well-recognized critical factors for the progression of HF $[1,2,6,12,13,16]$. We have shown that ACF TGR exhibit markedly enhanced HF-related mortality compared with that in ACF Hannover Sprague-Dawley rats (HanSD), i.e., transgene-negative normotensive controls $[8,30,37]$.

Given the advantages of the described experimental models and availability of 14,15EETs analog [disodium (S)-2-(13-(3-pentyl)ureido)-tridec-8(Z)-enamido)succinate, EET-A], which was previously found to be suitable for long-term in vivo studies [21,38-40], we first aimed to examine effects of chronic EET-A treatment on the morbidity and mortality in ACF TGR and compare it with the standard pharmacological blockade of the RAS with angiotensin-converting enzyme (ACE) inhibitor (ACEi), as described earlier [30,37,41].

In HF patients, the prognosis is worsened when the disease is accompanied by kidney dysfunction ("cardiorenal syndrome") [3,12,42-44]. Therefore, to gain a better insight into the possible role of interactions of CYP-derived eicosanoids with other vasoactive/neurohormonal systems in the pathophysiology of ACF-induced HF, kidney messenger ribonucleic acid (mRNA) expression analysis was performed, with a particular focus on the genes that were previously implicated in the pathophysiology of HF [12]. In addition, to explore in more detail the interactions of CYP-derived eicosanoids and RAS in the pathophysiology of ACF-induced HF, the concentrations of EETs, DHETEs, angiotensin II (ANG II), and angiotensin-1-7 (ANG 1-7) were measured. Moreover, since inappropriate activation of the sympathetic nervous system (SNS) is known to contribute to the progression of HF $[14,45,46]$, the concentrations of norepinephrine (NE) were also measured.

$\omega$-hydroxylase, another CYP-450-dependent enzyme of AA metabolism, generates hydroxyeicosatetraenoic acids (HETEs), mainly 20-HETE [17,47]. Since it might have some role in the progression of HF [48,49], we measured tissue 20-HETE concentrations in this study along with tissue protein expression of CYP2C23 and CYP2J3, the enzymes responsi- 
ble for EETs formation, and CYP4A1, the enzyme responsible for HETEs production [50]. Also measured was sEH, the enzyme which degrades EETs [7-10,17]. To obtain knowledge about the neurohormonal activity levels before initiating the treatment regimens, all the parameters mentioned above were assessed in sham-operated TGR, HanSD rats, and untreated ACF TGR two weeks after the ACF operation.

Moreover, to further elucidate the mechanism(s) underlying possible beneficial actions of EET-A on the course of ACF-induced HF, we assessed cardiac structure and function using echocardiography and invasive pressure-volume analysis of the left ventricle (LV). In addition, the renal clearance studies were performed in separate groups of animals. This was done after two weeks of treatment because at this stage untreated ACF TGR began systematically to die. Additionally, in another group of animals that survived until the end, we performed long-term observations (after 20 weeks) and the analyses analogous with those performed within the short-term protocol.

\section{Materials and Methods}

\subsection{Animals}

All animals used in the present study were bred at the Center for Experimental Medicine of this Institute from stock animals supplied by the Max Delbrück Center for Molecular Medicine, Berlin, Germany. Heterozygous TGR were generated by breeding male homozygous TGR with female homozygous HanSD rats as described in the original study [35], age-matched HanSD rats served as transgene-negative normotensive controls. The animals were kept on a 12-h/12-h light/dark cycle. Throughout the experiments, rats were fed a normal salt, normal protein $\operatorname{diet}(0.45 \% \mathrm{NaCl}, 19-21 \%$ protein) manufactured by SEMED (Prague, Czech Republic) and had free access to tap water.

\subsection{Heart Failure Model, Exclusion Criteria}

Eight-week-old male TGR rats were anesthetized with an intraperitoneal injection of ketamine/midazolam mixture (Calypsol, Gedeon Richter, Hungary, $160 \mathrm{mg} / \mathrm{kg}$ and Dormicum, Roche, France, $160 \mathrm{mg} / \mathrm{kg}$ ). Chronic HF due to volume overload was induced by creating ACF using a needle technique. This procedure is routinely performed in our laboratory, and the technique's details were reported previously $[26,31,32,37,51]$. Shamoperated rats underwent an identical procedure but without creating the ACF. Animals in which a technical error occurred during ACF creation or a pulsatile flow in the inferior vena cava could not be confirmed, suggesting flawed ACF function, were excluded from the study.

\subsection{Detailed Experimental Design}

2.3.1. Series 1: Assessment of Kidney mRNA Expression in the Early Phase of ACF-Induced HF

The aim was to evaluate the activation of the RAS, CYP-dependent pathways, and the SNS in the kidney at the mRNA level. The animals underwent sham-operation or ACF creation as described above and 14 days later were killed by decapitation. Kidney tissue samples were immediately harvested into liquid nitrogen and stored at $-80^{\circ} \mathrm{C}$ until analysis. The following experimental groups were examined ( $n=9$ in each group):

1. Sham-operated HanSD rats

2. Sham-operated TGR

3. ACF TGR

The gene expression was determined as described earlier [52]; the procedure is in everyday use in our laboratory [53]. In all experiments, relative gene expression was calculated by the $2-\Delta \Delta \mathrm{Ct}$ method, which is most frequently used for such experiments [52]. This method directly uses the $\mathrm{Ct}$ (threshold cycle) information generated from a qPCR system. To calculate relative gene expression in target and reference samples, we employed the 18S rRNA gene as a housekeeping gene and used it as the normalizer because its expression level remains relatively stable in response to any treatment $[52,53]$. 
First, we calculated $\Delta \mathrm{Ct}$ of each sample following the formula:

$$
\Delta \mathrm{Ct}=\mathrm{Ct} \text { (gene of interest) }-\mathrm{Ct} \text { (housekeeping gene) }
$$

The expression of mRNA of selected genes was related to that in a control group, i.e., sham-operated HanSD rats. The final results were expressed as the n-fold difference in gene expression of mRNA of target genes between the appropriate experimental group and control group calculated as follows:

$\mathrm{n}-$ fold expression $=2-(\Delta \mathrm{Ct}$ of the experimental group $-\Delta \mathrm{Ct}$ of the control group $)$

Subsequently, the log transformation of the data was performed to make it more symmetrical, as recommended and generally accepted for evaluation of relative gene expression results [52-55]. Thus, the values in the graphs represent $\log 2 \mathrm{n}$-fold gene expression. The measurement of multiple mRNA expressions was performed in accordance with the manufacturer's instructions (384-well microfluidics TaqMan array cards; custom setting of selected genes; Applied Biosystems, Foster City, CA, USA). The following investigating genes are presented below including the appropriate ID assay identification number and abbreviation given by the manufacturer. The following genes were analyzed in the kidney cortex (Table 1):

Table 1. Genes analyzed in the kidney cortex.

\begin{tabular}{|c|c|c|}
\hline ID Assay Number & Gene Name & Abbreviation \\
\hline Rn00561847_m1 & renin & Ren \\
\hline Rn00561094_m1 & angiotensin I converting enzyme & Ace \\
\hline Rn01416293_m1 & angiotensin I converting enzyme 2 & Ace2 \\
\hline Rn00593114_m1 & $\begin{array}{l}\text { angiotensinogen } \\
\text { (serpin peptidase inhibitor, clade A, member 8) }\end{array}$ & Agt \\
\hline Rn02758772_s1 & angiotensin II receptor, type $1 \mathrm{a}$ & Agtr1a \\
\hline Rn00562673_s1 & MAS1 proto-oncogene, G protein-coupled receptor & Mas1 \\
\hline Hs99999901_s1 & 18S rRNA ribosomal subunit & 18s rRNA \\
\hline Rn00598510_m1 & cytochrome P450, family 4 , subfamily a, polypeptide 1 & Cyp4a1 \\
\hline Rn01413752_m1 & cytochrome P450, family 2 , subfamily c, polypeptide 23 & Сур2c23 \\
\hline Rn00567876_m1 & adrenoceptor alpha $1 \mathrm{~A}$ & Adra1a \\
\hline Rn01471343_m1 & adrenoceptor alpha $1 \mathrm{~B}$ & Adra1b \\
\hline Rn00562488_s1 & adrenoceptor alpha $2 \mathrm{~A}$ & Adra2a \\
\hline Rn00593312_s1 & adrenoceptor alpha 2B & Adra2b \\
\hline Rn00593341_s1 & adrenoceptor alpha $2 \mathrm{C}$ & Adra2c \\
\hline Rn00824536_s1 & adrenoceptor beta 1 & Adrb1 \\
\hline Rn00560650_s1 & adrenoceptor beta 2 , surface & Adrb2 \\
\hline Rn00560677_s1 & angiotensin II receptor, type 2 & Agtr2 \\
\hline
\end{tabular}

2.3.2. Series 2: Assessment of RAS, SNS and CYP Metabolites and Enzymes in the Early Phase of ACF-Induced HF

The aim was to evaluate the degree of systemic (i.e., plasma) and intrarenal activation of two axes of the RAS and SNS. The second aim was to assess the degree of activation of CYP-450-dependent epoxygenase and $\omega$-hydroxylase pathways in the kidney and LV tissues.

Animals were exposed to the same protocol as described in series 1, and the same three experimental groups were evaluated ( $n=12$ in each group). 
Since it is now well recognized that ANG II and ANG 1-7 concentrations in anesthetized animals are higher than those measured in decapitated conscious rats and that normotensive animals exhibit a greater increase in renin secretion in response to anesthesia than observed for ANG II-dependent hypertensive internal renin-depleted animals $[36,55,56]$, at the end of experiments plasma and tissue samples were obtained without anesthesia, i.e., from rats that were killed by decapitation. ANG II and ANG 1-7 were determined by radioimmunoassay, and NE levels were measured by solid-phase enzyme-linked immunosorbent assay. The extracts for EETs, DHETEs, and 20-HETE were separated by reverse-phase high-performance liquid chromatography and analyzed by negative-mode electrospray ionization and tandem mass spectroscopy. In addition, Western blot analysis of protein expression of CYP4A1, CYP2C23, and CYP2J3 in the kidney cortex and LV tissue was performed. These methods are described in detail in our previous studies and are routinely employed in our laboratory $[31,32,36-40,53,56,57]$.

2.3.3. Series 3 and 6: Effects of Treatment with EET-A or ACEi, Alone or Combined, on the Survival Rate and Albuminuria, and Effects of 20-Weeks' Treatment on Basal Cardiac Function Assessed by Echocardiography and by Pressure-Volume Analysis

Male rats of the same age as in series 1 and 2 derived from numerous litters were randomly assigned to experimental procedures (sham-operation or ACF creation). The animals from a single litter did not prevail in any group. Animals underwent either shamoperation or ACF creation described above on the day labeled as -14 and were left without treatment for 14 days. On a day marked 0, animals that underwent ACF creation were randomly assigned to the specific experimental group. At this time point (day 0), the rats were assigned into the following experimental groups:

1. Sham-operated HanSD rats (initial $\mathrm{n}=20$ )

2. Sham-operated TGR (initial $\mathrm{n}=21$ )

3. $\mathrm{ACF}$ TGR + water (i.e., untreated) (initial $\mathrm{n}=32$ )

4. $\quad$ ACF TGR + EET-A (initial $n=32$ )

5. ACF TGR + ACEi (initial $n=32$ )

6. ACF TGR + EET-A + ACEi (initial $\mathrm{n}=33$ )

The follow-up period was until day +140 (20 weeks). On the days labeled $0,+14,+28$, +70 , and +140 , the animals were placed in metabolic cages, and 24-h urinary albuminuria was assessed as described previously [32]. To obtain reliable data regarding the effects of treatment regimens on the survival rate and to have a sufficient number of rats to analyze cardiac function and structure by echocardiography and LV pressure-volume analysis, relatively high initial $\mathrm{n}$ values were used (also in sham-operated animals). To define such required initial $n$ values, statistical power analysis by the method developed by Cohen [58] was used. In the end, in the survived animals, the cardiac function by echocardiography and subsequently by LV pressure-analysis were assessed as described in detail in our previous studies $[37,41,57,59]$. In sham-operated animals, twelve animals were randomly assigned for these evaluations.

2.3.4. Series 4: Effects of 2-Weeks' Treatment with EET-A and ACEi, Alone or Combined, on Basal Cardiac Function Assessed by Echocardiography and by

Pressure-Volume Analyses

Animals were prepared as in the previous series. On day 0, the pharmacological treatment was initiated and pursued for 14 days; then, the animals were anesthetized using sodium thiopental (50 $\mathrm{mg} / \mathrm{kg}$ body weight), and echocardiography and pressurevolume analysis were performed. The following experimental groups $(n=14$ in each) were evaluated

1. Sham-operated HanSD rats

2. Sham-operated TGR

3. ACF TGR + water

4. ACF TGR + EET-A 
5. $\mathrm{ACF} \mathrm{TGR}+\mathrm{ACEi}$

6. $\mathrm{ACF}$ TGR + EET-A + ACEi

2.3.5. Series 5: Effects of 2-Weeks' Treatment with EET-A and ACEi, Alone or Combined, on Renal Hemodynamics and Excretory Function

In this series, the following groups, subjected to the same protocol as in Series 4, were studied ( $\mathrm{n}=12$ in each group):

1. Sham-operated HanSD rats

2. Sham-operated TGR

3. ACF TGR + water

4. $\mathrm{ACF}$ TGR + EET-A

5. $\mathrm{ACF}$ TGR + ACEi

6. ACF TGR + EET-A + ACEi

At the end of the experiment $($ day +14$)$, rats were anesthetized using sodium thiopental (50 mg/kg body weight), and acute clearance experiments were performed as described in detail in our previous studies to determine renal hemodynamics and excretory parameters $[37,41,59,60]$.

\subsection{Statement of Ethics}

The study followed the guidelines and practices established by the Animal Care and Use Committee of IKEM, which accord with the national law and were approved by the Animal Care and Use Committee of the IKEM (March 2016) and, consequently, by the Ministry of Health of the Czech Republic (project decision 17124/2016-OZV-30.0-8.3.16/2).

\subsection{Statistical Analysis}

Statistical analysis of the data was performed using Graph-Pad Prism 7 (Graph Pad Software, San Diego, CA, USA). Comparison of survival curves was performed by the log-rank (Mantel-Cox) test followed by the Gehan-Breslow-Wilcoxon test. Statistical comparisons of all other results were made by one-way ANOVA. The values are expressed as the mean \pm SEM; $p<0.05$, indicating a statistically significant difference.

\section{Results}

3.1. Series 1 and 2: Assessment of Kidney mRNA Expression and RAS, SNS and CYP Active Agents, Metabolites and Enzymes in the Early Phase of ACF-Induced HF in the Early Phase of ACF-Induced HF

Results of these Series are summarized in Figure 1 and Supplemental Figures S1-S6. We did not observe significant differences in the kidney mRNA expression of analyzed enzymes and receptors in the early phase of ACF-induced HF. Only the expression of renin in the kidney was significantly reduced in sham-operated TGR and ACF TGR when compared to sham-operated HanSD (Supplementary Figure S1).

In sham-operated TGR, higher plasma and kidney ANG II levels were observed as compared with sham-operated HanSD rats. At the same time, the ANG 1-7 levels were on the same level in sham-operated TGR as in control HanSD rats, which resulted in the impaired systemic and intrarenal balance between vasodilator and vasoconstrictor axes of the RAS expressed as the ratio of ANG 1-7 to ANG II values (Supplementary Figure S2). In our previous studies [56,57], this ratio was validated as a reliable marker of the activity of the vasodilator axis of the RAS when the vasoconstrictor axis is hyperactive. Significantly lower values of this index were now observed in plasma and kidneys of sham-operated TGR compared with the sham-operated HanSD rats. Moreover, the creation of ACF significantly increased this index; in the kidney tissue, it reached the levels observed in sham-operated HanSD rats, and in the plasma, it was even higher (Supplemental Figure S2). 


\section{Basal values \\ (2 weeks after ACF induction, i.e. before start of treatment)}

A

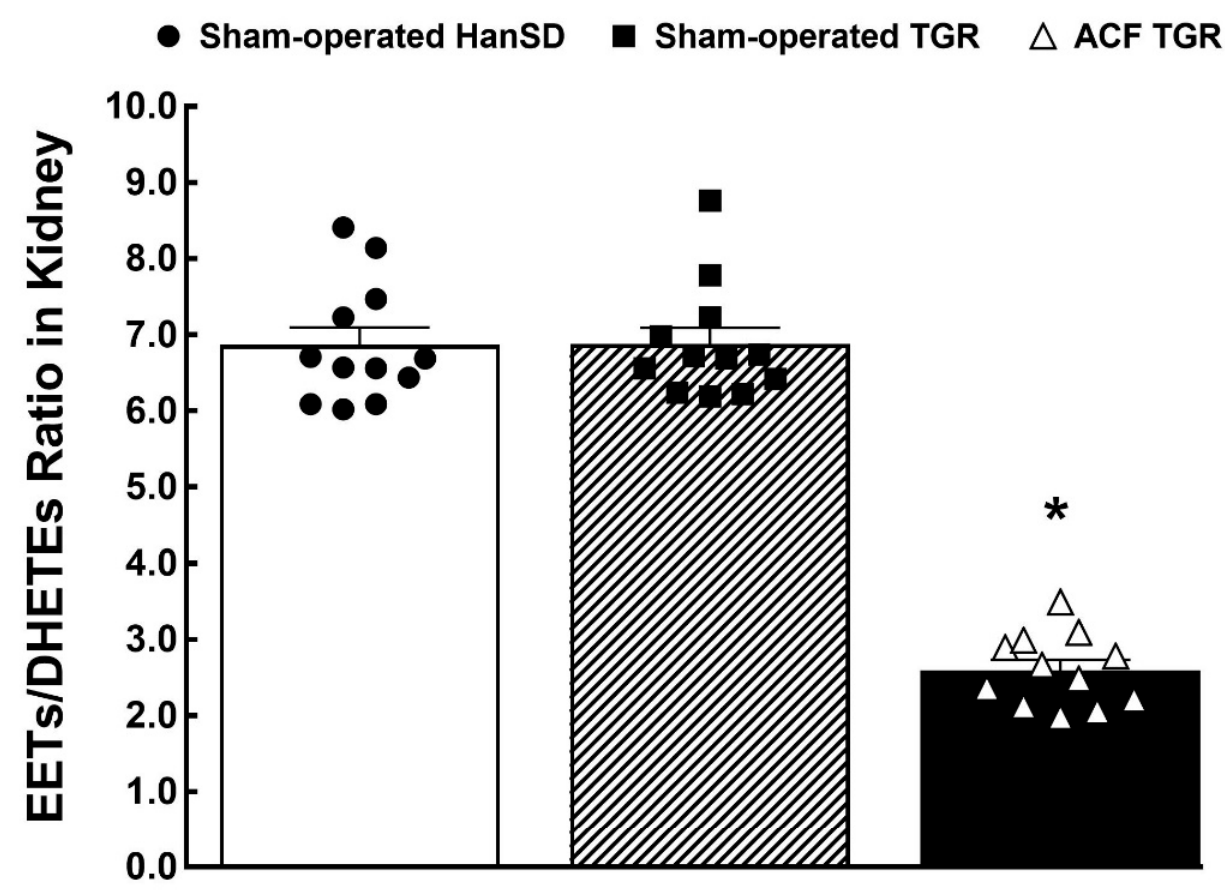

B

- Sham-operated HanSD Sham-operated TGR $\triangle$ ACF TGR

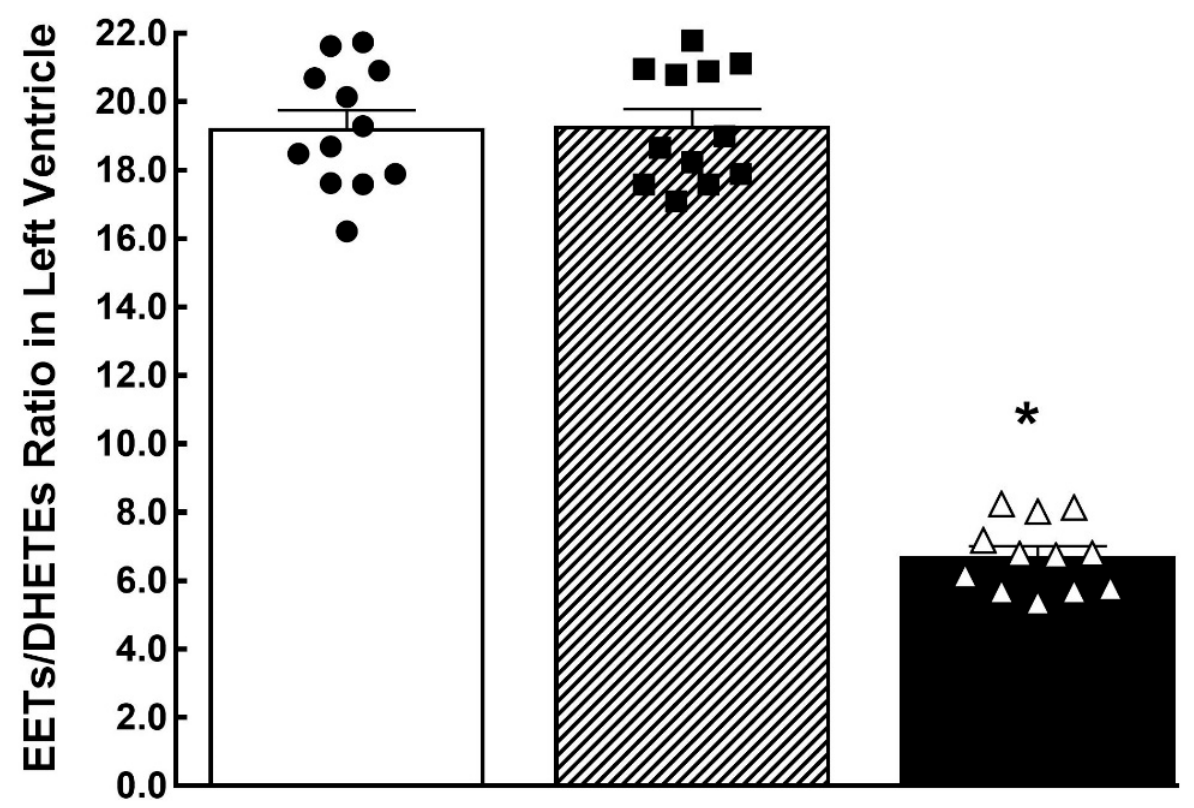

Figure 1. Assessment of kidney and left ventricle tissue availability of biologically active products of cytochrome P450-dependent epoxygenase pathway of arachidonic acid metabolism. Kidney (A) and left ventricle (B) ratio in epoxyeicosatrienoic acids (EETs) to dihydroxyeicosatrienoic acids (DHETEs) in sham-operated transgene-negative Hannover Sprague-Dawley rats (HanSD), sham-operated heterozygous Ren-2 transgenic rats (TGR), and TGR rats with aorto-caval fistula (ACF) two weeks after creation of ACF or sham-operation. ${ }^{*} p<0.05$ versus sham-operated HanSD rats and sham-operated TGR. 
With regards to the activation of the SNS, our results show that there were no significant differences in plasma and kidney NE levels among experimental groups (Supplementary Figure S3).

Furthermore, there were no significant differences in the concentration of 20-HETE and the expression of CYP4A1 protein in the kidney and LV tissues, which suggests that there were no differences in the CYP-450-dependent $\omega$-hydroxylase pathway of AA metabolism among all experimental groups (Supplementary Figure S4). Meanwhile, the deficiency in the CYP-dependent epoxygenase pathway of AA was observed in ACF TGR as compared with sham-operated TGR (Supplementary Figures S5 and S6 and Figure 1). The kidney and LV tissue deficiency in the biologically active epoxygenase products was revealed mainly in the 11,12-EET and 14,15-EET and it was accompanied by a significant rise of biologically inactive metabolites of CYP-dependent epoxygenase pathway of AA (DHETEs).

Decreased availability of biologically active epoxygenase products expressed as the EETs/DHETEs ratio is commonly used to assess the bioavailability of these metabolites [7-9] as was repeatedly validated also in our previous studies [37-41]. As summarized in Figure 1, this ratio was reduced by around 65\% in the kidney and LV in ACF TGR as compared with sham-operated TGR. Moreover, as shown in Supplemental Figure S4 there were no significant differences in the kidney and LV protein expression of the enzymes responsible for EETs production among experimental groups. The expression of $\mathrm{sEH}$ protein, an enzyme responsible for the conversion of EETs to DHETEs, was significantly increased by the creation of ACF in LV tissue (Supplemental Figure S4).

\subsection{Series 3: Effects of Single Treatment with EET-A or ACEi or with a Combination Thereof on the Survival Rate and Albuminuria}

All sham-operated HanSD rats and TGR (no ACF) survived until the end of the experiment (omitted for clarity). As shown in Figure 2, untreated ACF TGR began to die on day +3 (17 days after the creation of $A C F)$, and by day +70 , all the animals were dead. The treatment with EET-A and ACEi, alone or combined, markedly improved the survival rate, and the final rates were $45.5 \%$ (EET-A alone), 59.4\% (ACEi alone), and 71\% (EET-A and ACEi combined). However, the between-group differences were not significant (TGR ACF + EET-A vs. TGR ACF + ACEi, $p=0.25$; TGR ACF + EET-A vs. TGR ACF + EET-A + ACEi, $p=0.20$; TGR ACF + ACEi vs. TGR ACF + EET-A + ACEi, $p=0.83$; untreated TGR ACF vs. all treatment regimes, $p<0.05$; sham-operated TGR vs. all treatment regimens $p<0.05)$.

Figure 3 shows that at the start (14 days after sham-operation or creation of ACF, before initiation of treatment), the sham-operated TGR displayed about 20-fold higher albuminuria than the sham-operated HanSD rats. Interestingly, the creation of ACF at this stage caused a significant (about 2.5-fold) decrease in albuminuria. In sham-operated HanSD rats, albuminuria significantly increased throughout the experiment, parallel with the animals' age. Nevertheless, in the end, it was still significantly lower than in shamoperated TGR at the start of the experiment ( $3.9 \pm 0.2$ vs. $8.2 \pm 1.6 \mathrm{mg} / 24 \mathrm{~h}, p<0.05)$. In sham-operated TGR, albuminuria was modestly elevated till week 10 , but at the end of the study (week 20), it was markedly above the level seen before the start of the experiment $(45.1 \pm 4.1$ vs. $8.2 \pm 1.6 \mathrm{mg} / 24 \mathrm{~h}, p<0.05)$.

All treatment regimens further reduced albuminuria in ACF TGR, but the treatments with ACEi, alone or combined with EET-A, were more effective than EET-A alone. In fact, the values of albuminuria at the end of the study were even lower than in the sham-operated HanSD rats at the start of the study $(0.14 \pm 0.02$ and $0.13 \pm 0.02$ vs. $3.9 \pm 0.2 \mathrm{mg} / 24 \mathrm{~h}$, respectively; $p<0.05$ in both cases, Figure 3 ).

The progressive increase in albuminuria in normotensive rats is a natural phenomenon of aging and age-related end-organ damage and in hypertensive rats, it is a generally acknowledged marker of hypertension-related end-organ damage. We have seen this age-dependent progression in sham-operated normotensive, i.e., healthy animals, and in two models of hypertensive rats in our recent studies [32,40]. Nevertheless, it is important to recognize that in sham-operated HanSD rats even if the albuminuria increased with the age, is still minimal. However, the unexpected finding is that two weeks after the creation 
of ACF the albuminuria was markedly reduced in ACF TGR. This is an entirely surprising finding, especially regarding our recent study in Fawn-hooded hypertensive rats (FHH), a genetic model of spontaneous hypertension associated with chronic kidney diseases (CKD). In this model of high-output $\mathrm{HF}$, the induction of $\mathrm{ACF}$ resulted in a progressive rise in albuminuria. In addition, the gradual increase in albumin excretion after the induction of ACF was also found in the control strain, i.e., in Fawn-hooded low-pressure rats (FHL), that do not spontaneously develop CKD [32]. Our working hypothesis is that the decrease in albuminuria in TGR in response to ACF could be mediated by more pronounced initial renal hypoperfusion in ACF TGR as compared with ACF FHH. Fawn-hooded rats, in contrast to TGR, are only slightly hypertensive (systolic blood pressure around $145-150 \mathrm{mmHg}$ ), whereas systolic blood pressure in conscious TGR at this age is already $180-190 \mathrm{mmHg}$. Therefore, the conceivable decrease in renal perfusion pressure in ACF TGR could elicit puzzling beneficial effects on albuminuria. Nevertheless, it is only an assumption and further studies are necessary to address this surprising finding.
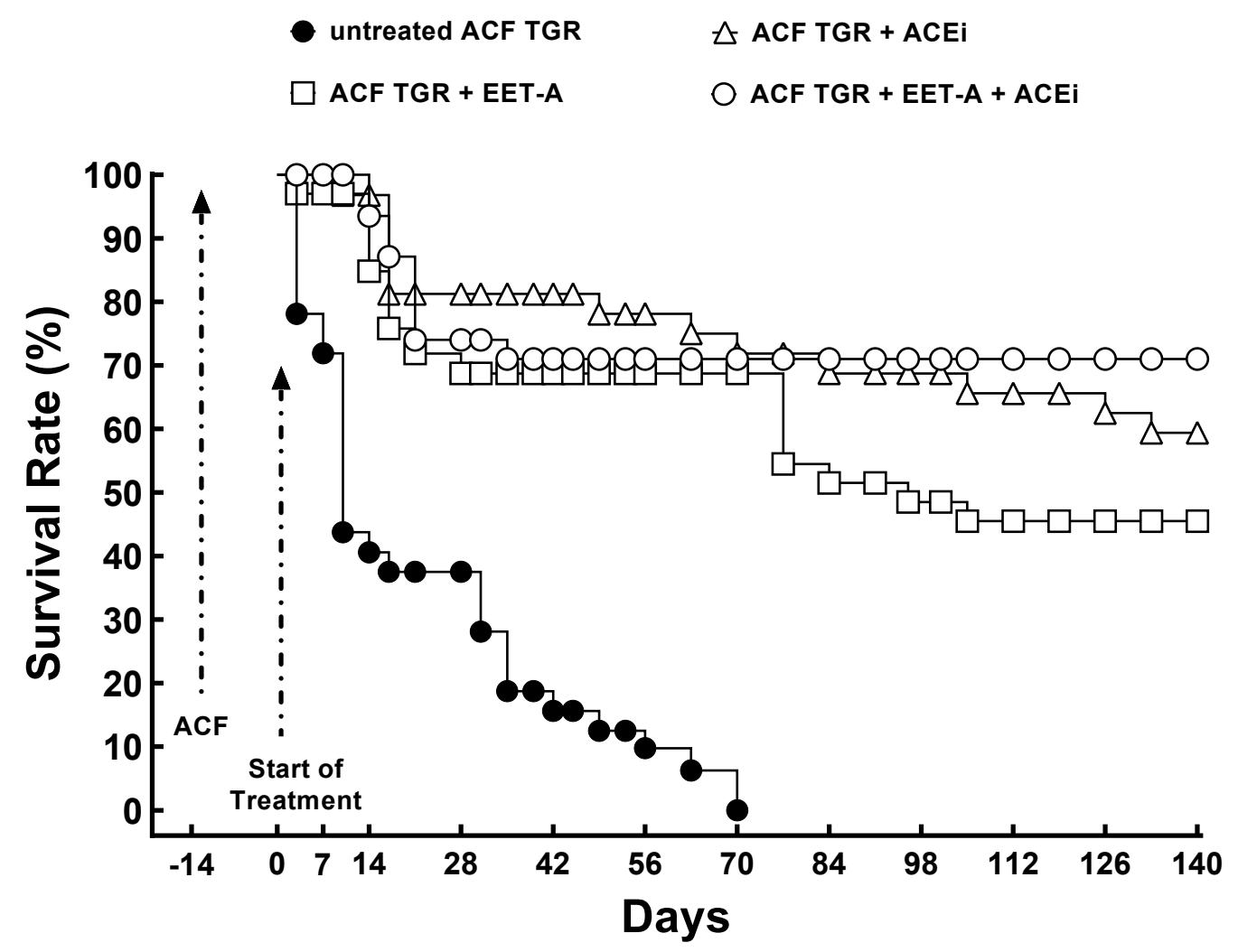

Figure 2. Assessment of the effects of treatment on the survival rate. The survival rate in sham-operated transgene-negative Hannover Sprague-Dawley (HanSD), sham-operated heterozygous Ren-2 transgenic rats (TGR), and TGR rats with aortocaval fistula (ACF) two weeks after the creation of ACF or sham-operation, treated either with angiotensin-converting enzyme inhibitor (ACEi) alone or with 14,15-epoxyeicosatrienoic acid analog (EET-A) alone or with the combination of EET-A and ACEi. 


$\begin{array}{lll}\square \text { Sham-operated HanSD } & \text { untreated ACF TGR } & \text { 日 ACF TGR + ACEi } \\ \square \text { Sham-operated TGR } & \text { 盂 ACF TGR + EET-A } & \text { \& ACF TGR + EET-A + ACEi }\end{array}$

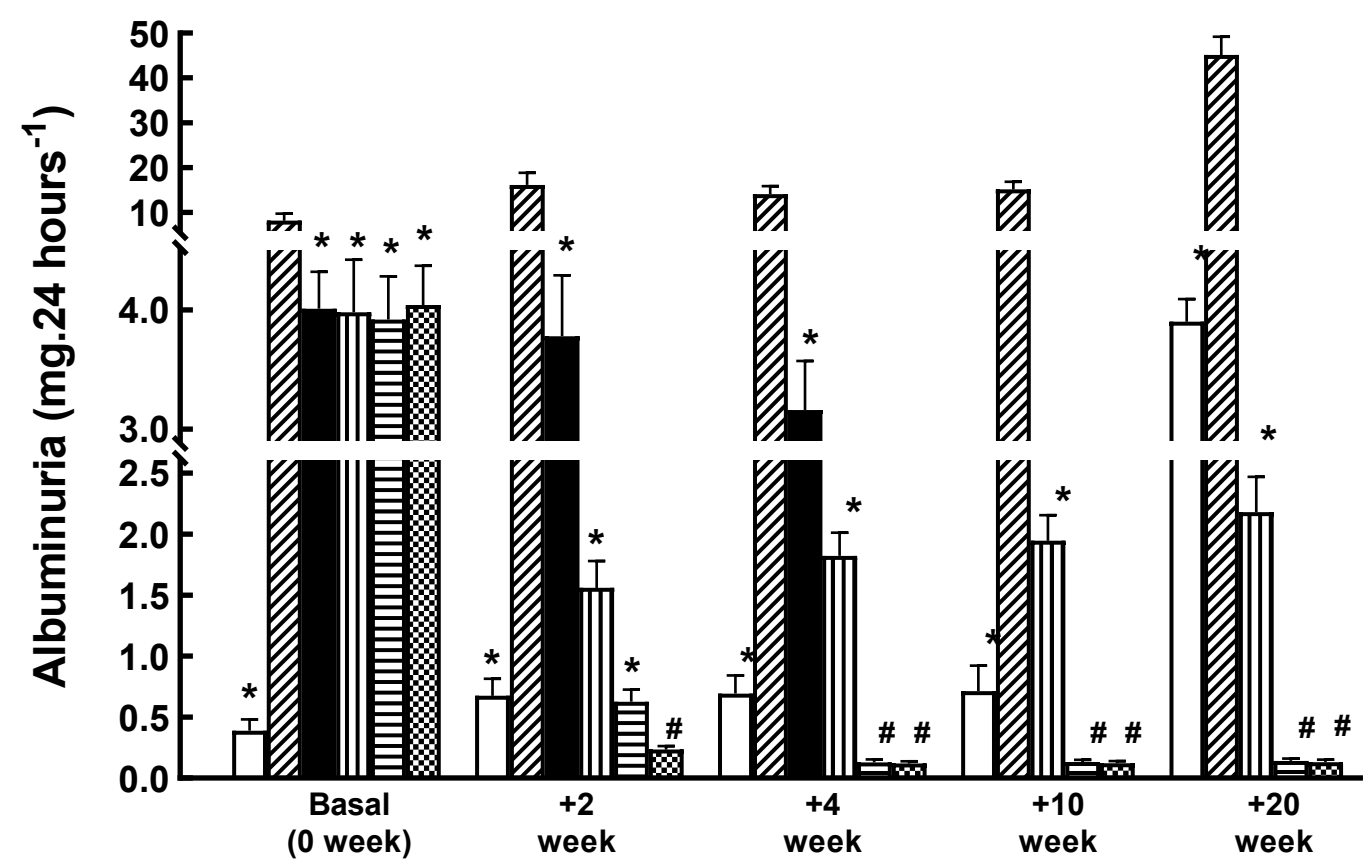

Figure 3. Assessment of the effects of treatment on albuminuria. Albuminuria in sham-operated transgene-negative Hannover Sprague-Dawley (HanSD), sham-operated heterozygous Ren-2 transgenic rats (TGR), and TGR rats with aortocaval fistula (ACF) two weeks after the creation of ACF or sham-operation, treated either with angiotensin-converting enzyme inhibitor (ACEi) alone or with 14,15-epoxyeicosatrienoic acid analog (EET-A) alone or with the combination of EET-A and ACEi. ${ }^{*} p<0.05$ versus sham-operated TGR. ${ }^{*} p<0.05$ versus sham-operated HanSD rats.

3.3. Series 4: Effects of 2-Weeks' Treatment with EET-A or ACEi, Alone or Combined, on Basal Cardiac Function Assessed by Echocardiography and by Pressure-Volume Analysis

Table 2 presents the body weight and organ weight parameters, factored by tibia length. The necessity of such correction is due to significant differences in body weight between untreated ACF TGR and sham-operated TGR, and the rationale for the factoring was documented in our previous work [61,62]. Also shown are the data for all ACF TGR groups exposed to various treatments, displaying remarkable if not significant body weight differences.

The data confirmed that sham-operated TGR displayed whole and LV cardiac hypertrophy as compared with sham-operated HanSD rats. Untreated ACF TGR demonstrated marked bilateral cardiac hypertrophy as seen from the normalized values of the whole heart weight, LV weight (with septum), and the right ventricle (RV) weight, as compared with the sham-operated TGR (no ACF). Moreover, untreated ACF TGR showed significantly higher lung weight than sham-operated TGR, suggesting substantial lung congestion. The treatment with EET-A alone did not alter any of the weight parameters in ACF TGR. In contrast, ACEi alone or combined with EET-A applied in ACF TGR significantly reduced, to a similar degree, the whole heart weight, LV and RV weights, and lung weight.

Table 3 summarizes the evaluation of cardiac function by echocardiography. There were no significant differences in the LV and RV diameters between sham-operated HanSD rats and sham-operated TGR. Nor were there significant differences in the LV and RV function (based on the LV ejection fraction, LV fractional shortening, RV fractional area change, and cardiac output) between sham-operated HanSD rats and sham-operated TGR. Notably, this evaluation corroborates that sham-operated TGR show increased LV anterior and posterior wall thickness and LV relative wall thickness as compared with sham-operated HanSD rats. Untreated ACF TGR exhibited increased stroke volume and cardiac output 
(the consequence of the shunt), markedly increased LV and RV diameters and decreased relative LV wall thickness (index of the development of eccentric hypertrophy). Also observed was significant impairment of the LV systolic function, as indicated by decreased $\mathrm{LV}$ fractional shortening and LV ejection fraction. This occurred without impairment of RV function, as seen from normal RV fractional area change, similar sham-operated TGR. The treatment with ACEi alone or EET-A alone did not change any of the parameters in ACF TGR, but the combined ACEi and EET-A treatment significantly reduced LV anterior and posterior wall thickness as compared with the untreated ACF TGR.

Table 2. Body and organ weights 4 weeks after creation of the aorto-caval fistula (ACF) or sham-operation and after 2 weeks' treatment with 14,15-epoxyeicosatrienoic acids analog (EET-A) and angiotensin-converting enzyme inhibitor (ACEi), alone or combined with EET-A.

\begin{tabular}{|c|c|c|c|c|c|c|}
\hline & Group & & & & & \\
\hline & HanSD & TGR & ACF TGR & ACF TGR & ACF TGR & ACF TGR \\
\hline & +water & +water & +water & $+\mathrm{ACEi}$ & +EET-A & +EET-A + ACEi \\
\hline Body Weight (g) & $465 \pm 7$ & $487 \pm 9$ & $415 \pm 9^{\#}$ & $460 \pm 16$ & $453 \pm 8$ & $449 \pm 7$ \\
\hline Tibia length (mm) & $38.2 \pm 0.3$ & $37.7 \pm 0.2$ & $37.5 \pm 0.2$ & $37.8 \pm 0.2$ & $37.3 \pm 0.3$ & $37.4 \pm 0.2$ \\
\hline Whole heart weight (mg)/tibia length (mm) & $37.69 \pm 0.74$ & $48.01 \pm 0.98^{*}$ & $68.27 \pm 1.07^{\#}$ & $54.49 \pm 1.06^{@}$ & $64.07 \pm 1.19$ & $56.69 \pm 1.04^{@}$ \\
\hline LV weight (mg)/tibia length (mm) & $24.56 \pm 0.21$ & $33.69 \pm 0.91 *$ & $40.79 \pm 0.89^{\#}$ & $33.86 \pm 0.24^{@}$ & $38.07 \pm 0.81$ & $35.76 \pm 0.26^{\circledR}$ \\
\hline RV weight (mg)/tibia length (mm) & $7.28 \pm 0.19$ & $7.56 \pm 0.21$ & $14.69 \pm 0.39^{\#}$ & $11.95 \pm 0.18^{\circledR}$ & $15.26 \pm 0.43$ & $11.69 \pm 0.21^{@}$ \\
\hline RV weight (mg)/LV weight (mg) & $0.296 \pm 0.02$ & $0.224 \pm 0.01$ & $0.361 \pm 0.03^{\#}$ & $0.353 \pm 0.02$ & $0.401 \pm 0.09$ & $0.327 \pm 0.02{ }^{\circledR}$ \\
\hline Lung weight (mg)/tibia length (mg) & $50.48 \pm 1.06$ & $48.52 \pm 1.29$ & $74.89 \pm 1.43^{\#}$ & $61.69 \pm 1.17^{\circledR}$ & $68.48 \pm 2.39$ & $56.64 \pm 1.23^{\circledR}$ \\
\hline Kidney weight (mg)/tibia length (mg) & $39.27 \pm 0.51$ & $42.19 \pm 1.29$ & $40.35 \pm 1.13$ & $36.94 \pm 1.44$ & $39.04 \pm 1.14$ & $37.89 \pm 0.71$ \\
\hline Liver weight (mg)/tibia length (mg) & $455 \pm 16$ & $459 \pm 17$ & $435 \pm 21$ & $449 \pm 24$ & $464 \pm 26$ & $432 \pm 29$ \\
\hline
\end{tabular}

Values are the means \pm SEM. LV, left ventricle; RV, right ventricle; ${ }^{*} p<0.05$ vs. sham-operated HanSD rats; ${ }^{*} p<0.05$ vs. TGR + water; (a) $p<0.05$ vs. ACF TGR + water.

Table 3. Echocardiographic analysis 4 weeks after the induction of aorto-caval fistula (ACF) or sham-operation and after 2 weeks' treatment with 14,15-epoxyeicosatrienoic acids analog (EET-A) and angiotensin-converting enzyme inhibitor (ACEi) alone or combined with EET-A.

\begin{tabular}{|c|c|c|c|c|c|c|}
\hline & Group & & & & & \\
\hline & HanSD & TGR & ACF TGR & ACF TGR & ACF TGR & ACF TGR \\
\hline & +water & +water & +water & $+\mathrm{ACEi}$ & +EET-A & +EET-A + ACEi \\
\hline Heart rate $\left(\mathrm{min}^{-1}\right)$ & $371 \pm 11$ & $376 \pm 10$ & $356 \pm 9$ & $368 \pm 8$ & $364 \pm 8$ & $354 \pm 11$ \\
\hline LV diastolic diameter (mm) & $6.64 \pm 0.13$ & $6.14 \pm 0.16$ & $9.99 \pm 0.24^{\#}$ & $8.95 \pm 0.21$ & $9.83 \pm 0.23$ & $10.27 \pm 0.25$ \\
\hline LV systolic diameter $(\mathrm{mm})$ & $3.39 \pm 0.16$ & $2.99 \pm 0.11$ & $6.06 \pm 0.27^{\#}$ & $5.36 \pm 0.21$ & $6.01 \pm 0.19$ & $6.37 \pm 0.21$ \\
\hline LV anterior wall thickness in diastole (mm) & $2.06 \pm 0.06$ & $2.76 \pm 0.04 *$ & $2.18 \pm 0.05^{\#}$ & $2.08 \pm 0.04$ & $2.06 \pm 0.05$ & $1.68 \pm 0.02^{\circledR}$ \\
\hline $\mathrm{LV}$ posterior wall thickness in diastole $(\mathrm{mm})$ & $2.22 \pm 0.06$ & $3.11 \pm 0.08 *$ & $2.47 \pm 0.08^{\#}$ & $2.39 \pm 0.06$ & $2.36 \pm 0.06$ & $1.87 \pm 0.02 @$ \\
\hline $\mathrm{LV}$ relative wall thickness & $0.67 \pm 0.02$ & $1.02 \pm 0.06^{*}$ & $0.48 \pm 0.02^{\#}$ & $0.54 \pm 0.03$ & $0.48 \pm 0.02$ & $0.37 \pm 0.02 @$ \\
\hline LV ejection fraction $(\%)$ & $78.2 \pm 1.7$ & $81.6 \pm 0.9$ & $67.2 \pm 1.1^{\#}$ & $66.8 \pm 1.3$ & $68.4 \pm 1.4$ & $65.2 \pm 1.2$ \\
\hline LV fractional shortening $(\%)$ & $48.5 \pm 1.7$ & $51.3 \pm 0.8$ & $39.6 \pm 1.1^{\#}$ & $40.3 \pm 1.1$ & $38.9 \pm 1.1$ & $37.6 \pm 0.9$ \\
\hline LV stroke volume $(\mu \mathrm{L})$ & $179 \pm 6.4$ & $156 \pm 9.9$ & $379 \pm 19.3^{\#}$ & $303 \pm 11$ & $364 \pm 18.4$ & $376 \pm 18.2$ \\
\hline Cardiac output (mL/min) & $60.9 \pm 1.7$ & $58.5 \pm 3.6$ & $133.6 \pm 5.5^{\#}$ & $111.5 \pm 4.6$ & $132.8 \pm 6.8$ & $139.5 \pm 6.1$ \\
\hline RV basal diameter in diastole (mm) & $3.23 \pm 0.08$ & $3.19 \pm 0.11$ & $5.61 \pm 0.36^{\#}$ & $4.26 \pm 0.26$ & $4.79 \pm 0.25$ & $5.62 \pm 0.18$ \\
\hline RV midcavity diameter in diastole (mm) & $3.07 \pm 0.09$ & $2.67 \pm 0.14$ & $5.01 \pm 0.43^{\#}$ & $3.88 \pm 0.24$ & $4.05 \pm 0.20$ & $5.28 \pm 0.16$ \\
\hline RV fractional area change (\%) & $54.7 \pm 1.9$ & $55.7 \pm 2.8$ & $50.3 \pm 2.9$ & $49.4 \pm 2.1$ & $51.5 \pm 3.2$ & $44.1 \pm 2.9$ \\
\hline
\end{tabular}

Values are the means \pm SEM. LV, left ventricle; RV, right ventricle; ${ }^{*} p<0.05$ vs. sham-operated HanSD rats; ${ }^{\#} p<0.05$ vs. TGR + water;

${ }^{\circledR} p<0.05$ vs. ACF TGR + water. 


\section{Effects of 2-weeks treatment (4 weeks after ACF induction at the onset of decompensated phase of HF)}

A

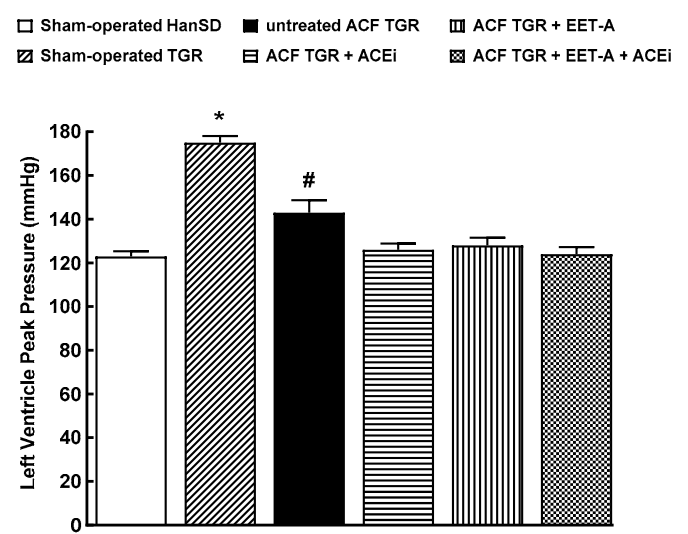

B

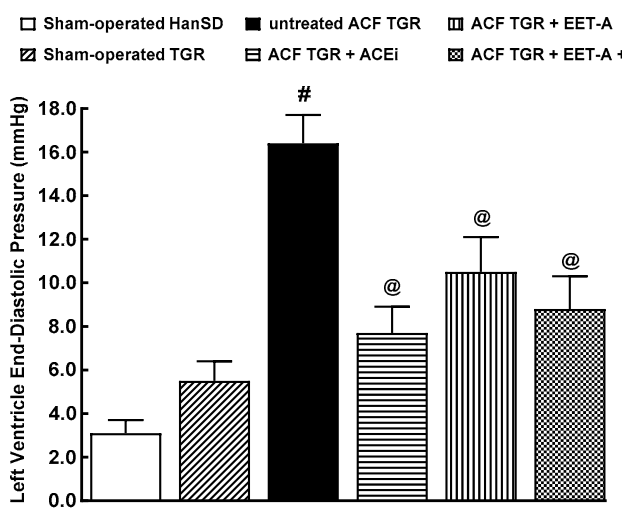

C

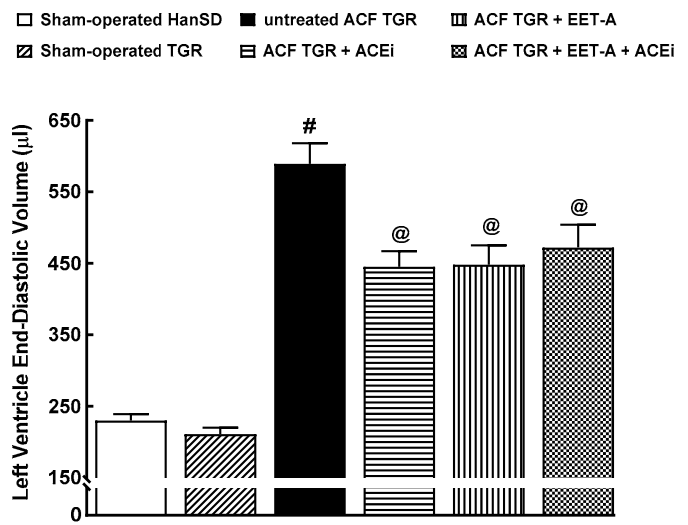

D

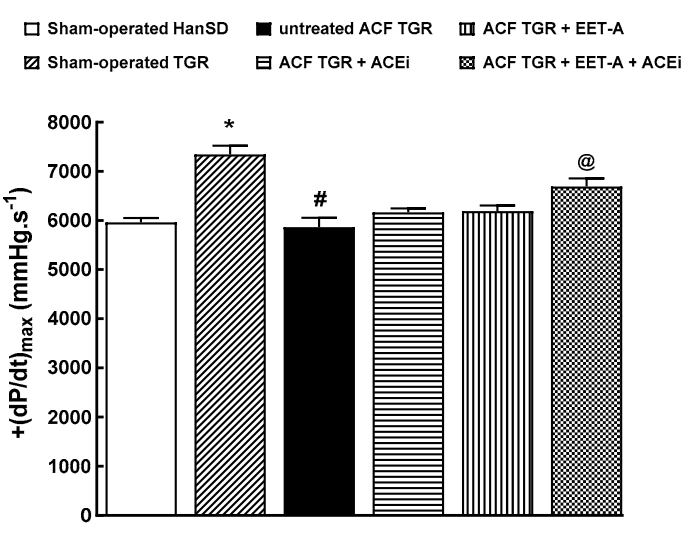

E

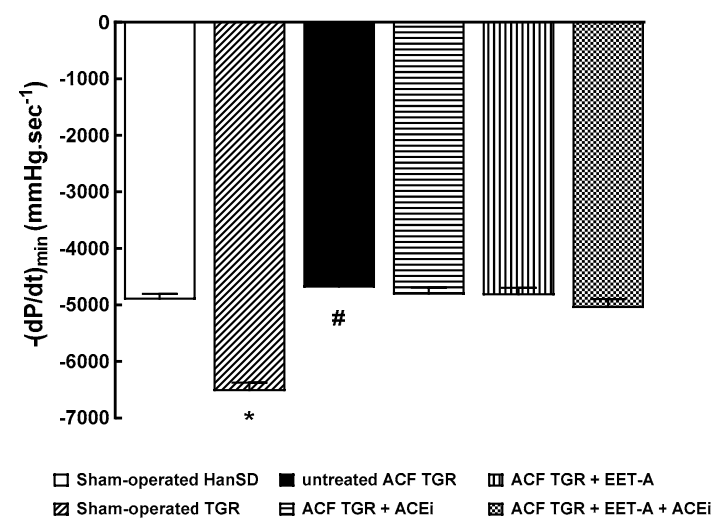

$\mathbf{F}$

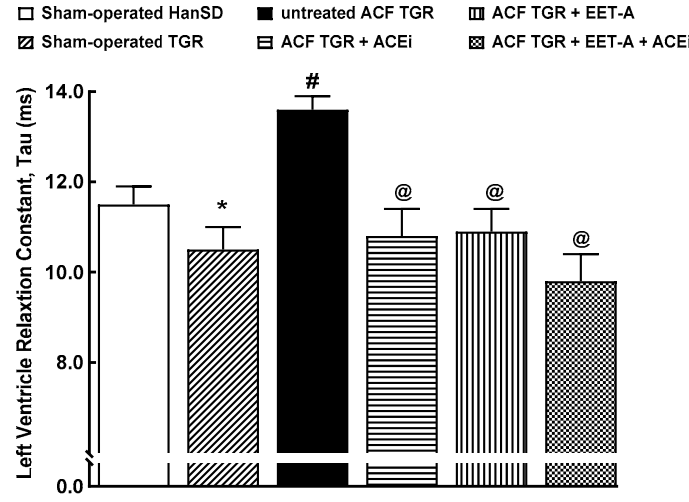

Figure 4. Part 1 of the left ventricular cardiac function assessment by invasive hemodynamic analysis performed 4 weeks after creation of the aorto-caval fistula (ACF) and 2 weeks after initiation of treatment in sham-operated transgene-negative Hannover Sprague-Dawley rats (HanSD), sham-operated heterozygous Ren-2 transgenic rats (TGR) and TGR rats with ACF, untreated or treated with angiotensin-converting enzyme inhibitor (ACEi) alone or with 14,15-epoxyeicosatrienoic acid analog (EET-A) alone or with the combination of EET-A and ACEi. Left ventricle peak pressure (A), left ventricle enddiastolic pressure (B), left ventricle end-diastolic volume $(\mathbf{C})$, maximum rates of pressure rise $(+\mathrm{dP} / \mathrm{dt})_{\max }(\mathbf{D})$, maximum rates of pressure fall $(-\mathrm{dP} / \mathrm{dt})_{\max }(\mathrm{E})$, relaxation constant tau $(\mathbf{F}) .{ }^{*} p<0.05$ sham-operated TGR versus sham-operated HanSD rats. ${ }^{\#} p<0.05$ for untreated ACF TGR versus sham-operated TGR. ${ }^{@} p<0.05$ versus untreated ACF TGR. 


\section{Effects of 2-weeks treatment \\ (4 weeks after ACF induction at the onset of decompensated phase of HF)}

A

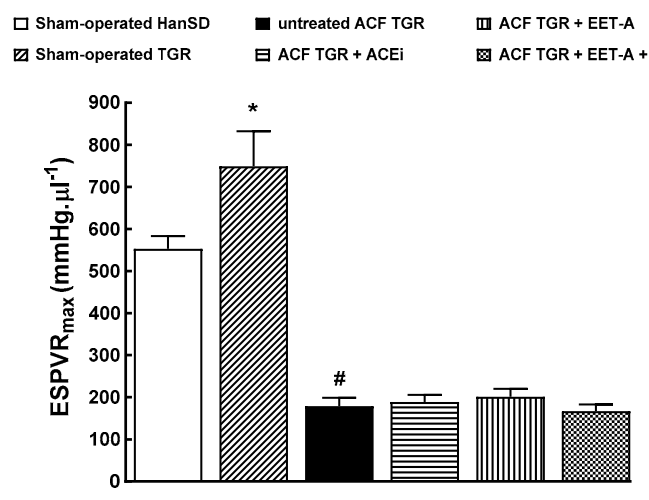

B
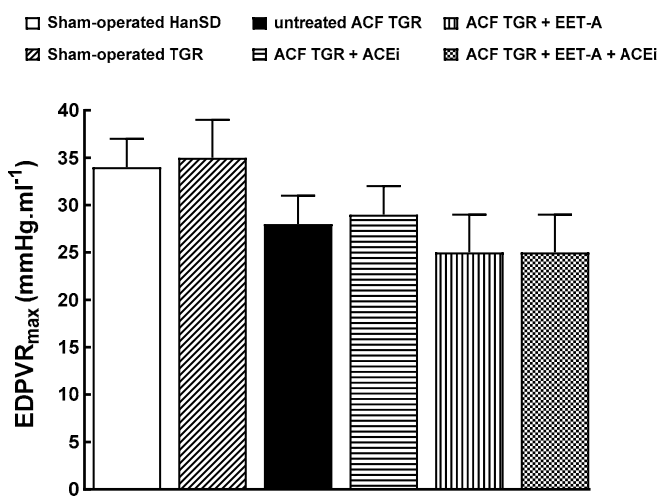

C
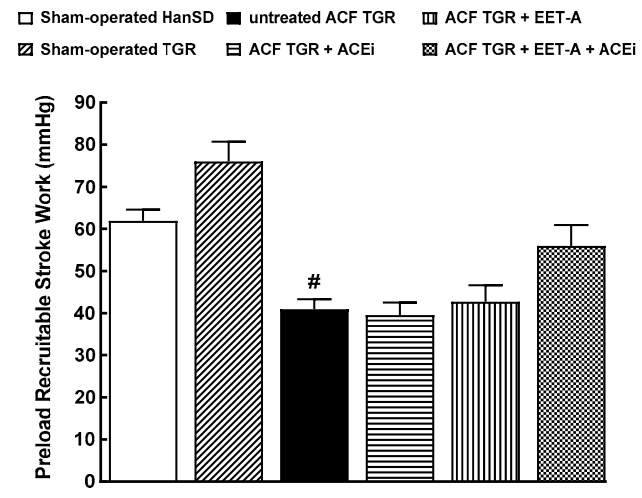

D

口 Sham-operated HanSD a untreated ACF TGR 四 ACF TGR + EET-A

v Sham-operated TGR $\quad$ ACF TGR + ACEi $\triangle$ ACF TGR + EET-A + ACE

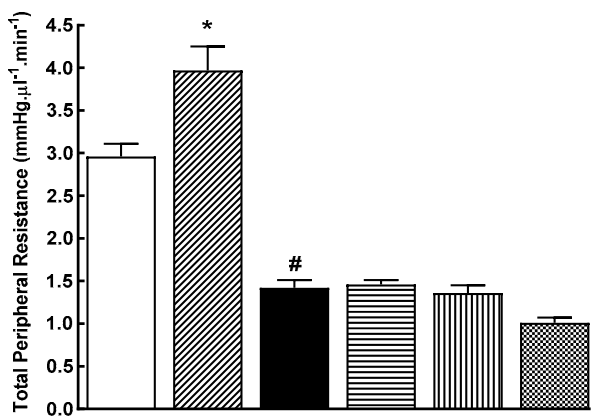

E
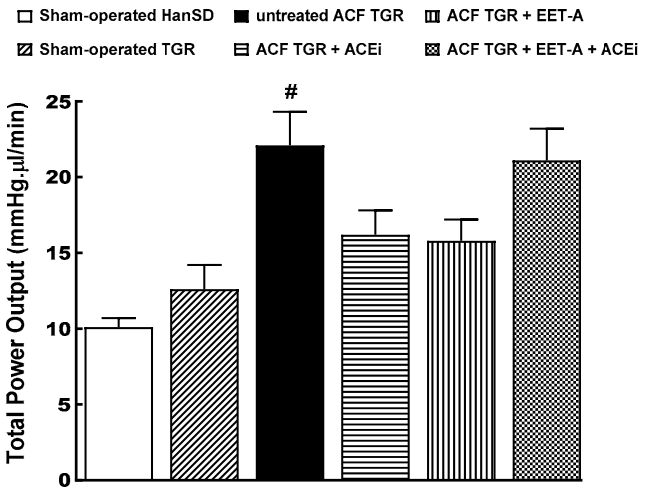

$\mathbf{F}$
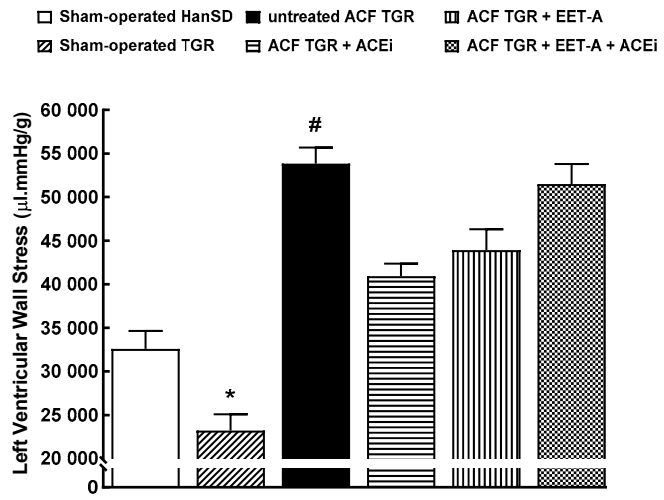

Figure 5. Part 2 of the left ventricular cardiac function assessment by invasive hemodynamic analysis performed 4 weeks after creation of the aorto-caval fistula (ACF) and 2 weeks after initiation of treatment in sham-operated transgene-negative Hannover Sprague-Dawley (HanSD), sham-operated heterozygous Ren-2 transgenic rats (TGR) and TGR rats with ACF, untreated or treated with angiotensin-converting enzyme inhibitor (ACEi) alone or with 14,15-epoxyeicosatrienoic acid analog (EET-A) alone or with combination of EET-A and ACEi. End-systolic pressure-volume relationship (ESPVR) (A), end-diastolic pressure-volume relationship (EDPVR) (B), preload recruitable stroke work (C), total peripheral resistance (D), total power output (E), left ventricular wall stress (F). ${ }^{*} p<0.05$ for sham-operated TGR versus sham-operated HanSD rats. ${ }^{\#} p<0.05$ for untreated ACF TGR versus sham-operated TGR. 
Figures 4 and 5 summarize the evaluation of cardiac function by the invasive hemodynamics method. Sham-operated TGR displayed higher LV peak pressure and maximum rates of pressure rise $(+\mathrm{dP} / \mathrm{dt}$ )max (Figure $4 \mathrm{~A}, \mathrm{D})$ and lower maximum rates of pressure fall $(-\mathrm{dP} / \mathrm{dt}) \mathrm{max}$ and of relaxation constant tau (Figure $4 \mathrm{E}, \mathrm{F})$ as compared with shamoperated HanSD rats, but there were no significant differences in LV end-diastolic pressure and volume between sham-operated animal groups (Figure 4B,C). Untreated ACF TGR displayed significant decreases in $\mathrm{LV}$ peak pressure, $(+\mathrm{dP} / \mathrm{dt}) \mathrm{max}$ and $(-\mathrm{dP} / \mathrm{dt}) \mathrm{max}$, as compared with sham-operated TGR. None of the treatment regimens attenuated these decreases (Figure 4A,D,E). In addition, untreated ACF TGR exhibited marked increases in $\mathrm{LV}$ end-diastolic pressure, LV end-diastolic volume, and LV relaxation constant tau, as compared with the sham-operated TGR, and all of the applied treatments (EET-A and ACEi, alone or combined) significantly attenuated these changes (Figure $4 B, C, F$ ).

Sham-operated TGR showed significantly elevated end-systolic pressure-volume relationship (ESPVR) and total peripheral resistance (Figure 5A,D), but also significantly lower LV wall stress (Figure 5F). Untreated ACF TGR displayed substantially decreased ESPVR, the preload recruitable stroke work (PRSW), and total peripheral resistance (TPR) (Figure 5A-D). On the other hand, they showed markedly increased total power output and LV wall stress (Figure 5E,F). The treatment with EET-A and ACEi, alone or combined, did not significantly change any of the parameters in ACF TGR.

\subsection{Series 5: Effects of 2-Weeks' Treatment with EET-A or ACEi, Alone or Combined, on Renal Hemodynamics and Excretory Function}

Untreated ACF TGR displayed lower mean arterial pressure (MAP) than shamoperated TGR, comparable with sham-operated HanSD rats (Figure 6A). The treatment with EET-A alone or ACEi alone or in combination significantly lowered MAP in ACF TGR, to a similar extent in each of the three groups. There were no significant between-group differences in the glomerular filtration rate (GFR) among all experimental groups (Figure 6B). Untreated ACF TGR exhibited renal blood flow (RBF) significantly lower than in shamoperated TGR (almost 40\% reduction). The treatment with EET-A alone or ACEi alone or in combination did not significantly change RBF in ACF TGR (Figure 4C). Untreated ACF TGR displayed substantially lower urine flow and absolute and fractional sodium excretion than sham-operated TGR. None of the treatments normalized these parameters in ACF TGR (Figure 6E,F).

\subsection{Series 6: Effects of 20-Weeks' Treatment with EET-A or ACEi, Alone or Combined, on Basal Cardiac Function Assessed by Echocardiography and by Pressure-Volume Analyses}

Table 4 summarizes body and organ weights in animals that survived until the end of the study (long-term observation). The degree of the whole and LV cardiac hypertrophy in the ACF TGR treated with ACEi alone was similar as observed in untreated ACF TGR in the early phase (i.e., four weeks after the creation of ACF). Moreover, the RV cardiac hypertrophy was even more pronounced in ACF TGR treated with ACEi at the end of the experiment when compared with untreated ACF TGR in the early phase (see Table 2). Likewise, the ACF TGR treated with EET-A alone showed a similar pattern in cardiac hypertrophy. These results indicate that the cardiac hypertrophy progressed in the animals that were treated with ACEi alone or EET-A alone in the long-term observation. Noteworthy is that the combined treatment with ACEi and EET-A significantly reduced the bilateral cardiac hypertrophy and lung congestion in ACF TGR when compared with solitary ACEi or EET-A.

Table 5 presents the evaluation of cardiac function in the animals that survived until the end of the experiment. All the ACF TGR treated groups showed markedly increased LV diameters that were even higher than those measured in the untreated ACF TGR four weeks after creating ACF. In contrast to the early phase, all ACF TGR groups displayed significant impairment of RV systolic function as indicated by decreased RV fractional area change. 


\section{Effects of 2-weeks treatment (4 weeks after ACF induction at the onset of decompensated phase of HF)}

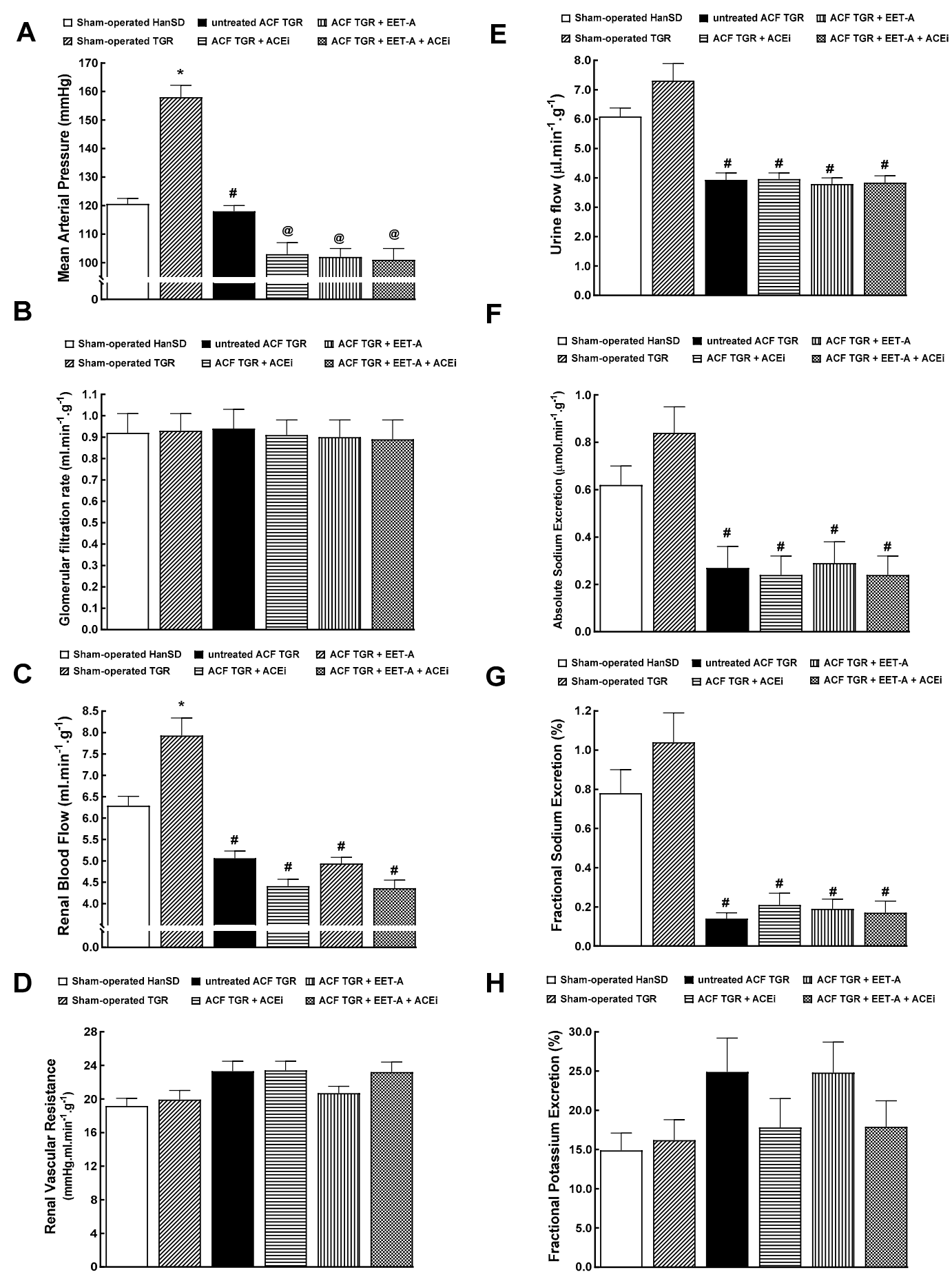

Figure 6. Renal function analysis was performed 4 weeks after the creation of the aorto-caval fistula (ACF) and 2 weeks after initiation of treatment in sham-operated transgene-negative Hannover Sprague-Dawley (HanSD), sham-operated heterozygous Ren-2 transgenic rats (TGR), and TGR rats with ACF, untreated or treated with angiotensin-converting enzyme inhibitor (ACEi) alone or with 14,15-epoxyeicosatrienoic acid analog (EET-A) alone or with the combination of EET-A and ACEi. Mean arterial pressure (A), glomerular filtration rate (B), renal blood flow (C), renal vascular resistance (D), urine flow (E), absolute (F) and fractional sodium excretion (G) and fractional potassium excretion $(\mathbf{H}){ }^{*} p<0.05$ for sham-operated TGR versus sham-operated HanSD rats. ${ }^{\#} p<0.05$ versus sham-operated TGR. ${ }^{@} p<0.05$ versus untreated ACF TGR. 
Table 4. Body and organ weights 22 weeks after creation of the aorto-caval fistula (ACF) or sham-operation and after 20-weeks' treatment with 14,15-epoxyeicosatrienoic acids analog (EET-A) and angiotensin-converting enzyme inhibitor (ACEi), alone or combined with EET-A.

\begin{tabular}{|c|c|c|c|c|c|}
\hline & Group & & & & \\
\hline & HanSD & TGR & ACF TGR & ACF TGR & ACF TGR \\
\hline & +water & +water & $+\mathrm{ACEi}$ & + EET-A & +EET-A + ACEi \\
\hline Body Weight (g) & $630 \pm 10$ & $633 \pm 9$ & $689 \pm 8^{\#}$ & $701 \pm 19$ & $608 \pm 18^{@}$ \\
\hline Tibia length (mm) & $43.8 \pm 0.2$ & $43.7 \pm 0.3$ & $44.1 \pm 0.4$ & $44.2 \pm 0.4$ & $44.1 \pm 0.4$ \\
\hline Whole heart weight (mg)/tibia length (mm) & $33.67 \pm 0.58$ & $46.69 \pm 0.79 *$ & $65.09 \pm 1.12^{\#}$ & $66.12 \pm 1.222^{\#}$ & $55.01 \pm 1.09 @$ \\
\hline LV weight (mg)/tibia length (mm) & $26.94 \pm 0.23$ & $34.55 \pm 0.89 *$ & $39.22 \pm 0.27^{\#}$ & $40.27 \pm 0.29^{\#}$ & $33.33 \pm 0.21^{@}$ \\
\hline RV weight (mg)/tibia length (mm) & $7.47 \pm 0.21$ & $7.57 \pm 0.18$ & $15.87 \pm 0.35^{\#}$ & $15.77 \pm 0.33^{\#}$ & $11.36 \pm 0.22^{@}$ \\
\hline RV weight (mg)/LV weight (mg) & $0.277 \pm 0.03$ & $0.219 \pm 0.03$ & $0.405 \pm 0.04^{\#}$ & $0.392 \pm 0.03^{\#}$ & $0.341 \pm 0.03 @$ \\
\hline Lung weight (mg)/tibia length (mg) & $49.77 \pm 1.18$ & $48.06 \pm 1.23$ & $61.91 \pm 1.51^{\#}$ & $66.97 \pm 1.78^{\#}$ & $51.29 \pm 0.98^{@}$ \\
\hline Kidney weight (mg)/tibia length (mg) & $42.69 \pm 0.99$ & $42.56 \pm 1.17$ & $39.01 \pm 1.23$ & $40.27 \pm 1.16$ & $39.23 \pm 1.25$ \\
\hline Liver weight (mg)/tibia length (mg) & $458 \pm 19$ & $440 \pm 23$ & $444 \pm 27$ & $437 \pm 25$ & $447 \pm 28$ \\
\hline
\end{tabular}

Values are the means \pm SEM. $L V$, left ventricle; RV, right ventricle; ${ }^{*} p<0.05$ vs. sham-operated HanSD rats; ${ }^{\#} p<0.05$ vs. TGR + water; ${ }^{\circledR} p<0.05$ vs. ACF TGR + ACEi and vs. ACF TGR + EET-A.

Table 5. Echocardiographic analysis 22 weeks after the induction of aorto-caval fistula (ACF) or sham-operation and after 20 weeks' treatment with 14,15-epoxyeicosatrienoic acids analog (EET-A) and angiotensin-converting enzyme inhibitor (ACEi) alone or combined with EET-A.

\begin{tabular}{|c|c|c|c|c|c|}
\hline & Group & & & & \\
\hline & HanSD & TGR & ACF TGR & ACF TGR & ACF TGR \\
\hline & +water & +water & $+\mathrm{ACEi}$ & +EET-A & +EET-A + ACEi \\
\hline Heart rate $\left(\min ^{-1}\right)$ & $372 \pm 9$ & $365 \pm 18$ & $363 \pm 9$ & $365 \pm 11$ & $368 \pm 19$ \\
\hline LV diastolic diameter (mm) & $6.96 \pm 0.22$ & $7.09 \pm 0.27$ & $11.29 \pm 0.31^{\#}$ & $11.12 \pm 0.51^{\#}$ & $11.51 \pm 0.29^{\#}$ \\
\hline LV systolic diameter (mm) & $3.78 \pm 0.22$ & $4.35 \pm 0.39$ & $7.55 \pm 0.26^{\#}$ & $7.63 \pm 0.44^{\#}$ & $7.69 \pm 0.37^{\#}$ \\
\hline $\mathrm{LV}$ anterior wall thickness in diastole (mm) & $2.32 \pm 0.06$ & $3.05 \pm 0.06 *$ & $2.06 \pm 0.05^{\#}$ & $2.25 \pm 0.06^{\#}$ & $1.87 \pm 0.05^{\#}$ \\
\hline LV posterior wall thickness in diastole $(\mathrm{mm})$ & $2.55 \pm 0.06$ & $3.07 \pm 0.11^{*}$ & $2.08 \pm 0.05^{\#}$ & $2.12 \pm 0.07^{\#}$ & $2.02 \pm 0.05^{\#}$ \\
\hline LV relative wall thickness & $0.738 \pm 0.04$ & $0.913 \pm 0.04 *$ & $0.368 \pm 0.02^{\#}$ & $0.393 \pm 0.02^{\#}$ & $0.369 \pm 0.03^{\#}$ \\
\hline LV ejection fraction (\%) & $75.8 \pm 1.9$ & $71.6 \pm 2.6$ & $55.8 \pm 1.6^{\#}$ & $53.9 \pm 1.4^{\#}$ & $57.3 \pm 1.5^{\#}$ \\
\hline LV fractional shortening (\%) & $46.1 \pm 1.7$ & $44.6 \pm 1.6$ & $33.3 \pm 0.7^{\#}$ & $32.5 \pm 1.1^{\#}$ & $32.4 \pm 0.8^{\#}$ \\
\hline LV stroke volume $(\mu \mathrm{L})$ & $191 \pm 11$ & $174 \pm 8$ & $437 \pm 22 \#$ & $408 \pm 29^{\#}$ & $414 \pm 31 \#$ \\
\hline Cardiac output (mL/min) & $69.9 \pm 1.5$ & $63.6 \pm 4.2$ & $158 \pm 6.2^{\#}$ & $152 \pm 10.1^{\#}$ & $155 \pm 8.7^{\#}$ \\
\hline RV basal diameter in diastole (mm) & $3.06 \pm 0.13$ & $3.08 \pm 0.11$ & $6.65 \pm 0.19^{\#}$ & $5.81 \pm 0.36^{\#}$ & $5.41 \pm 0.13^{@}$ \\
\hline RV midcavity diameter in diastole (mm) & $2.45 \pm 0.11$ & $2.49 \pm 0.12$ & $6.03 \pm 0.16^{\#}$ & $5.28 \pm 0.21 \#$ & $5.02 \pm 0.12^{@}$ \\
\hline RV fractional area change (\%) & $61.9 \pm 5.8$ & $65.1 \pm 5.2$ & $35.9 \pm 1.1^{\#}$ & $39.5 \pm 1.6^{\#}$ & $33.7 \pm 1.4^{\#}$ \\
\hline
\end{tabular}

Values are means \pm SEM. LV, left ventricle; RV, right ventricle; ${ }^{*} p<0.05$ vs. sham-operated HanSD rats; ${ }^{*} p<0.05$ vs. TGR + water; ${ }^{\circledR} p<0.05$ vs. ACF TGR + ACEi.

Figures 7 and 8 summarize an evaluation of the cardiac function by the invasive hemodynamics method in animals that survived until the end of the study. At the late stage, the LV end-diastolic pressure in ACF TGR treated with ACEi or EET-A was similar as found in untreated ACF TGR in the early phase (compare Figures 4B and 7B), but the combined treatment with EET-A and ACEi significantly reduced it to the level observed in sham-operated animals (Figure 7B). Moreover, in all the ACF TGR that survived until the end of the experiment, the LV end-diastolic volume was even higher than observed in untreated ACF TGR in the early phase (Figures $4 \mathrm{C}$ and $7 \mathrm{C}$ ), and none of the treatment regimens reduced it (the combined treatment tended to decrease it, NS). 


\section{Effects of 20-week treatment (22 weeks after induction of ACF, animals that survived until end of study)}

A

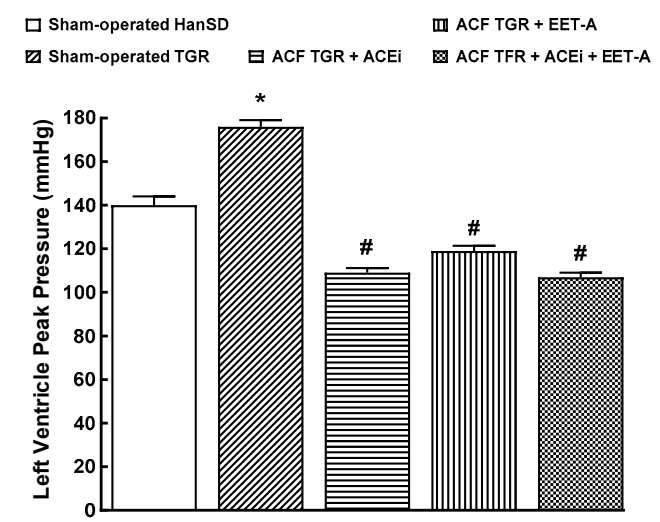

B

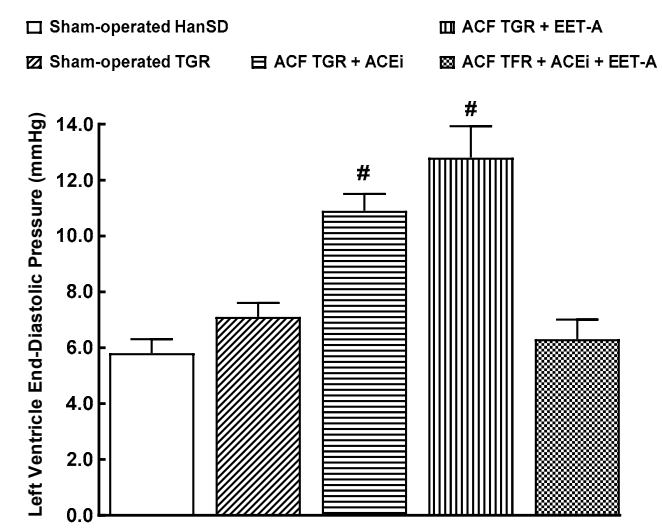

C

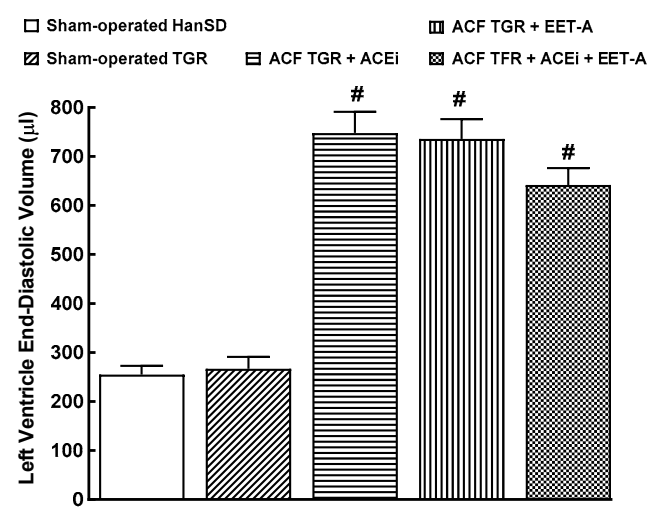

D

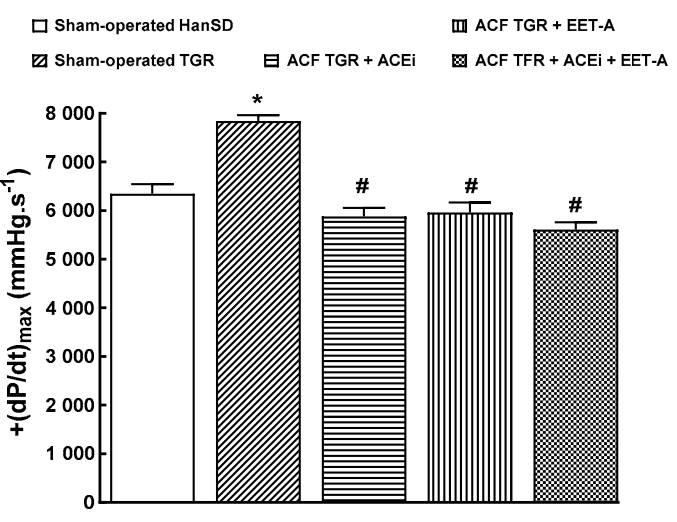

E

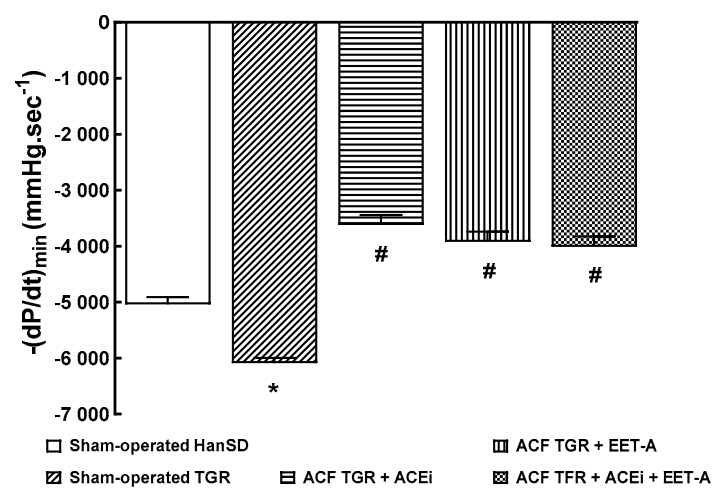

$\mathbf{F}$

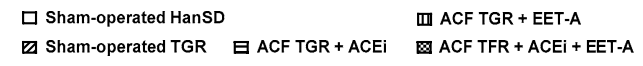

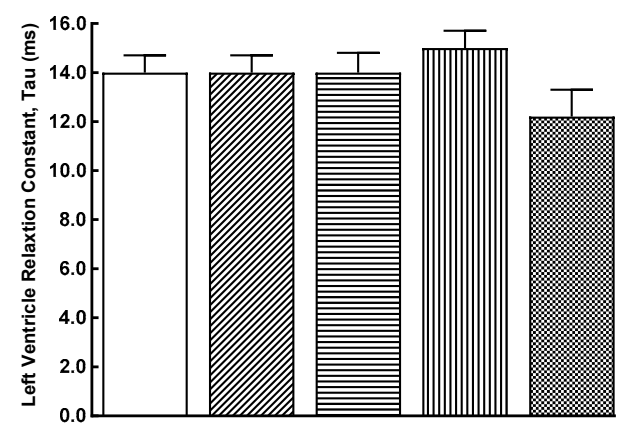

Figure 7. Part 1 of the left ventricular cardiac function assessment by invasive hemodynamic analysis performed 22 weeks after creation of the aorto-caval fistula (ACF) and 20 weeks after initiation of treatment in sham-operated transgene-negative Hannover Sprague-Dawley (HanSD), sham-operated heterozygous Ren-2 transgenic rats (TGR) and TGR rats with ACF treated with angiotensin-converting enzyme inhibitor (ACEi) alone or with 14,15-epoxyeicosatrienoic acid analog (EET-A) alone or with the combination of EET-A and ACEi. Left ventricle peak pressure (A), left ventricle end-diastolic pressure (B), left ventricle end-diastolic volume $(\mathbf{C})$, maximum rates of pressure rise $(+\mathrm{dP} / \mathrm{dt})_{\max }(\mathbf{D})$, maximum rates of pressure fall $(-\mathrm{dP} / \mathrm{dt})_{\max }(\mathrm{E})$, relaxation constant tau $(\mathbf{F}) .{ }^{*} p<0.05$ for sham-operated TGR versus sham-operated HanSD rats. ${ }^{\#} p<0.05$ for ACF TGR versus sham-operated TGR. 


\section{Effects of 20-week treatment (22 weeks after induction of ACF, animals that survived until end of study)}

A
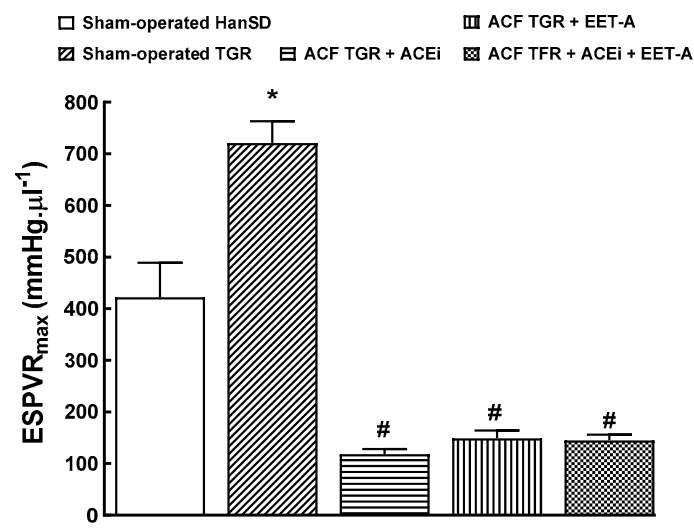

B

$\square$ Sham-operated HanSD Z Sham-operated TGR

W $\mathrm{ACF} T \mathrm{TR}+\mathrm{EET}-\mathrm{A}$

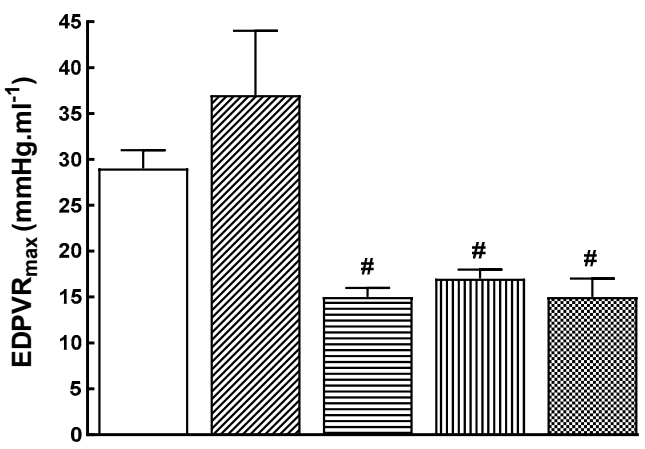

C

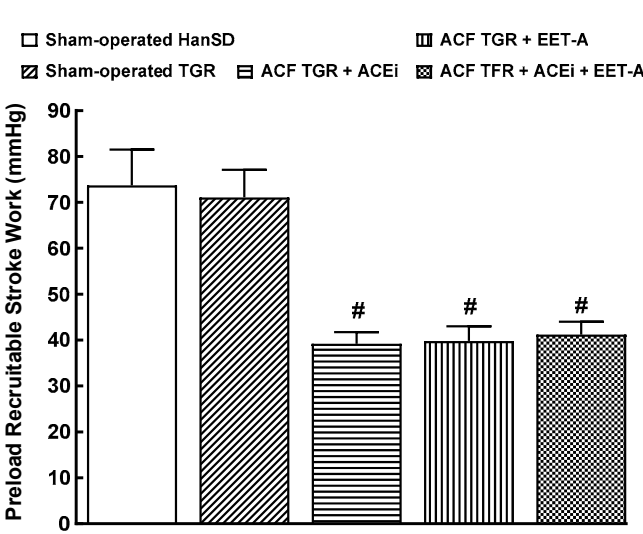

D

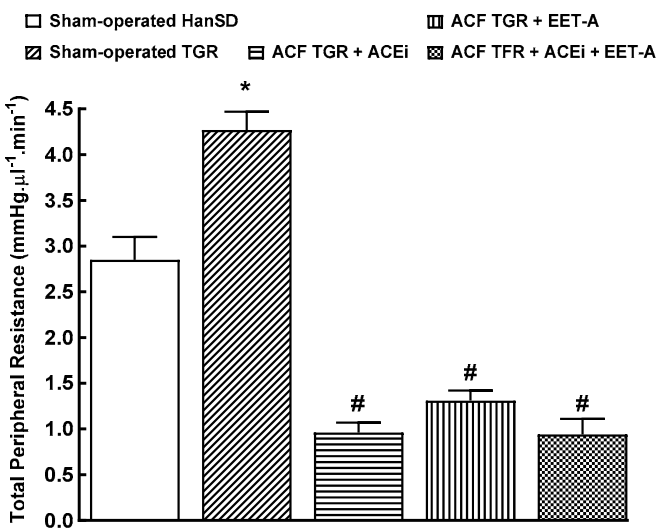

E

$\square$ Sham-operated HanSD

$\checkmark$ Sham-operated TGR $\boxminus$ ACF TGR + ACEi

II ACF TGR + EET-A

M ACF TFR + ACEi + EET-A

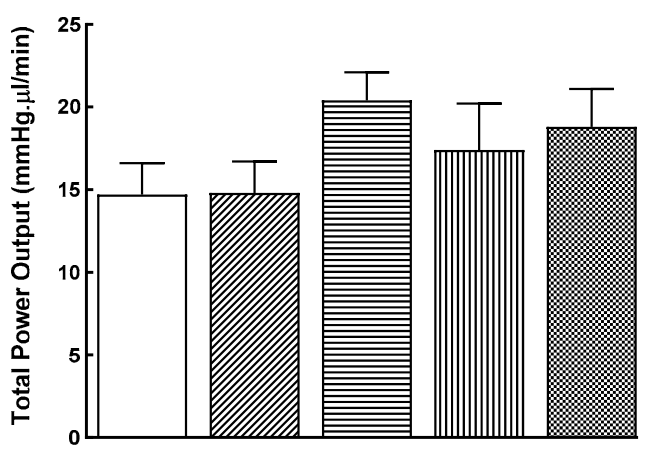

$\mathbf{F}$

ש Sham-operated TGR $\quad$ ACF TGR + ACEi $\triangle$ ACF TFR + ACEi + EET-A

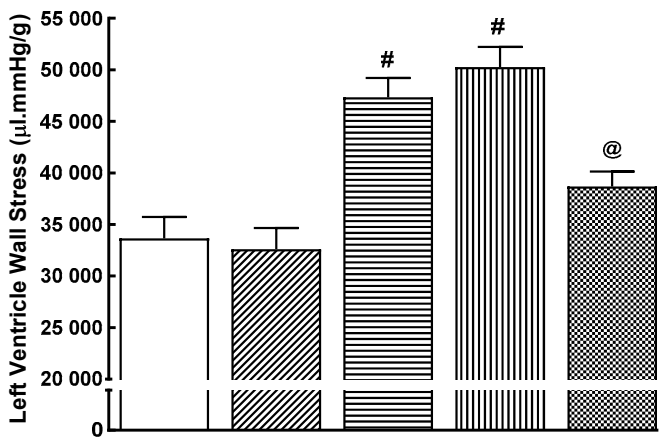

Figure 8. Part 2 of the left ventricular cardiac function assessment by invasive hemodynamic analysis performed 22 weeks after creation of the aorto-caval fistula (ACF) and 20 weeks after initiation of treatment in sham-operated transgene-negative Hannover Sprague-Dawley (HanSD), sham-operated heterozygous Ren-2 transgenic rats (TGR) and TGR rats with ACF, untreated or treated either with angiotensin-converting enzyme inhibitor (ACEi) alone or with 14,15-epoxyeicosatrienoic acid analog (EET-A) alone or with the combination of EET-A and ACEi. End-systolic pressure-volume relationship (ESPVR) (A), end-diastolic pressure-volume relationship (EDPVR) (B), preload recruitable stroke work (C), total peripheral resistance (D), total power output (E), left ventricular wall stress (F). ${ }^{*} p<0.05$ sham-operated TGR versus sham-operated HanSD rats. \# $p<0.05$ for ACF TGR versus sham-operated TGR. ${ }^{@} p<0.05$ versus other ACF TGR. 
All ACF TGR groups showed decreased ESPVR, EDPVR, PRSW, and TPR compared with the sham-operated TGR (Figure 8A-D). ACF TGR treated with ACEi or EET-A displayed increased LV wall stress compared with the sham-operated TGR, but the combined treatment with EET-A and ACEi significantly reduced it to the level that was not significantly different from that in the sham-operated TGR (Figure 8F).

\section{Discussion}

\subsection{Activity of the Neurohormonal Systems in ACF-Induced Heart Failure}

The degree of activation of the vasoactive/neurohormonal systems was evaluated in the very early phase of the ACF-induced HF model (two weeks after the ACF creation, i.e., just before the treatment regimens were initiated). Our data indicate that in this ANG II-dependent model of hypertension, the creation of ACF leads to the activation of the intrarenal and systemic vasodilatory /natriuretic axis of the RAS (based on ANG 1-7 concentrations and ANG 1-7/ ANG II ratio). There were no significant differences in plasma and intrarenal NE concentrations, suggesting no evident activation of the systemic and intrarenal SNS two weeks after the creation of ACF. Thus, the tentative conclusion is that at this very early phase, the major neurohormonal systems, i.e., vasoconstrictor axis of the RAS and SNS were not activated, in contrast to their long-lasting activation and deleterious actions, which was previously described as neurohormonal theory on the progression of HF [14,15,63-65]; however, the counter-regulatory axis of the RAS is markedly activated in ACF TGR.

Given the recent discovery that elevated ANG 1-7/ANG II ratio predicts a beneficial outcome of HF [66], our present findings are significant in that they suggest that the initial compensatory response in the ACF-induced model of HF in TGR consists of the activation of the vasodilatory / natriuretic axis of the RAS, which may override the effects of activation of the vasoconstrictor/sodium retaining axis. At first glance, these findings seem to diverge from our previous studies in ACF TGR [37,51,67], in which we observed that plasma and kidney ANG II, aldosterone, and NE levels are much higher than in their sham-operated counterparts. Thus, in this HF model, the vasoconstrictor/sodium retaining axis of the RAS along with the sympathorenal axis appeared to be activated. The reason for this discrepancy might be that our earlier studies were performed five weeks after the creation of ACF [37]. This was done by analogy with the protocol used in normotensive rats, which after five weeks, develop an early, compensated phase HF $[28,59,60,68]$. However, such selection of the study time proved to be inappropriate in TGR: we demonstrated that the course of high-output HF in TGR is dramatically accelerated [31,37,41,51] when compared both with normotensive and even with spontaneously hypertensive rats (SHR) [69]. In TGR, five weeks after the creation of the ACF, already 50\% mortality is observed, compared to simultaneous $100 \%$ survival rate in normotensive rats and SHR. Therefore, in the present study, the treatment was initiated earlier (two weeks after ACF), at the stage when 100\% survival was still observed. Moreover, our present biochemical data regarding the RAS and SNS show that ACF TGR were indeed in HF's early compensation phase.

Interestingly, kidney mRNA expression of the rat renin gene was markedly suppressed in sham-operated as well as ACF TGR, which is in accordance with the earlier TGR studies $[35,70,71]$. Furthermore, it was reported that murine Ren-2 mRNA is expressed at very high levels, especially in the adrenal glands $[70,71]$. The suppression of the kidney native rat renin gene is mediated through a well-known negative feedback inhibition [72] Thus, our present findings confirm the consistency of our methods, which is particularly important with mRNA analyses in ACF TGR.

In addition, even though there were no significant between-group differences in kidney mRNA expression of enzymes responsible for the generation and degradation of EETs, our tissue protein expression and biochemical data unequivocally show that kidney and LV tissue availability of EETs in ACF TGR are reduced. This is not the consequence of decreased EETs formation (unaltered protein expressions of CYP2C23 and CYP2J3), but rather the result of increased conversion of EETs to DHETEs, as indicated by increased 
tissue sEH protein expression in these animals. These findings further support our view that the regulation and activity of neurohormonal/vasoactive systems is a very complex issue and any solid conclusions cannot be made based on the sole mRNA and/or protein analysis. The complexity of these systems requires a comprehensive analysis at all levels and at least measurements of biologically active peptides of the appropriate system.

Furthermore, our results show that renal and LV activity of the CYP-450-dependent $\omega$-hydroxylase pathway of AA metabolism is not increased in ACF TGR, based on unaltered CYP4A1 mRNA and protein expression and 20-HETE tissue concentration. These findings are important because it was proposed that under certain conditions, increased 20-HETE levels can importantly contribute to pathological cardiac hypertrophy, cardiac remodeling, and consequently to the progression of HF [48,49]. Our present findings in ACF TGR do not support this hypothesis. Thus, the second tentative conclusion is that in the early phase, ACF TGR have marked tissue deficiency of biologically active EETs. It has been noticed that EETs importantly protect the myocardium against deleterious cardiac remodeling [73], which is the main factor in the pathogenesis of HF [6,74]. Moreover, they exhibit important renoprotective actions [7-9]. Considering our recent findings that ACF TGR display reduced renal vasodilatory responses to EETs $[67,75]$, all of the abovementioned factors support the rationale for treating ACF TGR with EET-A. Furthermore, our data show the dominant role of the tissue deficiency of the 14,15-EETs, in agreement with the accepted knowledge that this isomer is crucial among CYP-dependent epoxygenase metabolites $[10,17,19,50]$. All this strengthens the basis for employing EET-A in the treatment of ACF TGR.

\subsection{Effects of the Treatment Regimens on the Survival Rate}

We found that the treatment with EET-A alone and ACEi alone considerably attenuated the dramatically aggravated HF-related mortality in ACF TGR. This accords well with our original hypothesis (supported by our comprehensive analyses of CYP-derived metabolites in kidney and LV tissue) that pharmacological enhancement of EETs actions should attenuate HF in ACF TGR, which might have substantial implications for the treatment of HF. Likewise, the beneficial effects of ACEi treatment on the survival rate accord with our line of reasoning: it is generally agreed that even if activation of the vasoconstrictor/sodium retaining axis of the RAS may be initially beneficial, the long-lasting actions might be detrimental and importantly contribute to the progression of HF to the fatal end $[6,14,15,63,64]$. In addition, in previous studies, we clearly demonstrated that the activity of circulating and intrarenal vasoconstrictor/sodium retaining axis of the RAS is markedly increased in ACF TGR in the advanced HF phase [37,51], and that blockade of this axis by ACEi dramatically improves survival rate $[30,37,41]$.

Collectively, the results demonstrate that both treatments effectively delay the HFrelated mortality in ACF TGR, even though each regimen affects specifically only one of the two vasoactive systems. This observation encouraged us to propose that the combined treatment with EET-A and ACEi should enhance the beneficial effects. However, this has not been corroborated: the protection against HF-related mortality was not improved.

Nevertheless, it is emphasized that the final survival rate in the group exposed to the combined treatment with EET-A and ACEi was numerically higher than in groups treated with either drug alone, though, admittedly, the difference did not reach statistical significance. Moreover, the combined treatment displayed some beneficial actions on cardiac morphology and function when evaluated after 20-weeks' treatment, such as more distinct effects, on the whole, LV and RV cardiac hypertrophy and lung congestion, and also attenuated RV dilatation, in agreement with the data on the organ weight and echocardiography. Furthermore, the pressure-volume analysis of cardiac function at this stage revealed that the combined treatment significantly reduced LV end-diastolic pressure and LV wall stress compared to the treatment with EET-A or ACEi alone. Evidently, with prolonged therapy, the additive beneficial actions on the course of HF-related mortality became apparent. Nevertheless, comprehensive long-term studies with a follow-up period 
of at least 60 weeks are necessary to draw definite conclusions. Moreover, for reliable analysis of the difference between the relevant survival curves, the initial $\mathrm{n}$ values should be at least 42 per group $[42,43]$. Obviously, such thorough studies are needed even though appropriate prolonged experiments would be difficult and time- and cost-consuming.

\subsection{Mechanisms of the Beneficial Actions of the Treatments}

A search for the mechanism(s) underlying the beneficial actions of each of the treatment regimens on HF-related mortality in ACF TGR was carried out after 2-weeks' treatment (i.e., four weeks after the creation of ACF) because at this time point untreated ACF TGR were showing strikingly high mortality (only $41 \%$ were alive, see the data of series 3$)$. Each treated group showed an almost complete survival rate $(85 \%, 97 \%$ and $94 \%$, respectively), which suggested that four weeks post-ACF was the onset point of the decompensation phase of HF in untreated ACF TGR. If so, the beneficial actions of the treatment regimens should be the most effective at this stage.

While the clinical term "cardiorenal syndrome" is a simplification as it does not encompass a spectrum of disorders involving the heart and the kidney [12], there is no doubt that the development of renal dysfunction predicts poor outcomes in patients with HF [3,42-44]. We showed previously that ACF TGR seems to be an optimal model for evaluating cardiorenal interaction in the pathophysiology of HF [31,37,51,67]. Moreover, it was revealed that some beneficial impact on the course of high-output HF in ACF TGR can be achieved by preventing renal dysfunction, e.g., by pharmacological blockade of sEH or AT1 receptors [37,51]. However, it was reported that when the pharmacological blockade of RAS by ACE inhibitor (ACEi) was employed in ACF TGR, the protective effect was dominantly mediated by attenuation of cardiac hypertrophy [51]. On the other hand, some authors reported that the development of cardiac hypertrophy after ACF induction is resistant to ACEi treatment [76]; however, this conclusion was based on the studies with normotensive animals with initially normal RAS activity. In addition, the response to the pharmacological blockade of RAS regarding the development of cardiac hypertrophy was reported to be dependent on the phase of cardiac hypertrophy after ACF induction [77]. Considering all these aspects, we have decided to evaluate renal as well as cardiac function at this critical phase to find out if the beneficial actions are achieved predominantly by renal or by cardiac mechanism(s) or by combination thereof.

We found that untreated ACF TGR displayed marked impairment of renal hemodynamics and renal excretory function, in agreement with previous studies in this model [31, $37,41,51,67]$, further supporting the notion that renal dysfunction importantly contributes to the reduced long-term survival rate in this high-output HF model. However, in contrast to our previous study [37], we did not find beneficial effects of ACEi treatment, alone or combined with EET-A, on RBF and renal excretion. Nor were such effects seen with EET-A alone. This was unexpected and somehow discouraging. However, as already discussed above, it is emphasized that beneficial actions of ACEi were seen in the study where the treatment was commenced five weeks after the creation of ACF [37] and lasted for additional five weeks, so it means the beneficial effects of ACEi on renal function were observed in animals that were actually in the very late phase of HF, principally ten weeks after induction of ACF. Thus, the effect of the treatment was explored in untreated ACF TGR in the very late phase of HF. As mentioned in our previous study [37], the major limitation of such protocol is that untreated ACF TGR exhibit almost $50 \%$ mortality at this time point, therefore the assessments were performed in an extremely selected population of ACF TGR, which was probably the most resistant subpopulation of animals to the development of high-output HF. To avoid this drawback, our present study was also performed in the early phase of HF. This explanation should reconcile our present and previous findings concerning the effect of treatments with ACEi either alone or in combination with EET-A on renal function in ACF TGR. However, the present finding is unexpected because it indicates that renal mechanisms are not dominantly responsible for the beneficial effects of all the treatment regimens in this study. In this context, it is worth reminding our recent study in 
which renal denervation improved the survival rate in ACF TGR; however, the beneficial effect was not associated with any improvement of reduced RBF [31]. This is puzzling because it is believed that removing the deleterious influence of renal sympathetic nerve activity, which is the hallmark of HF $[14,15,45,46]$, should improve impaired RBF. This further underscores the complexity of the role of renal dysfunction in the pathophysiology of HF.

Concerning cardiac morphology and cardiac function, we have found that the creation of ACF in TGR resulted after four weeks in marked eccentric chamber remodeling and cardiac hypertrophy, related to the enhanced cardiac output dependent on blood recirculation through the fistula. Load-dependent and load-independent parameters of LV contractility [impaired $+(\mathrm{dP} / \mathrm{dt})$ max, suppressed ESPVR, lower PRSW, as well as decreased LV ejection fraction and LV fractional shortening] indicate distinct systolic dysfunction in this very early phase of HF. In addition, the decreased - (dP/dt)max and increased LV relaxation constant indicate impairment of diastolic function in untreated ACF TGR. Simultaneously, untreated ACF TGR exhibited a marked increase in LV end-diastolic pressure and volume accompanied by a pronounced increase in the lung but not liver weight. Taken together, these findings indicate that four weeks after ACF creation, the untreated ACF TGR displayed marked systolic and diastolic dysfunction with signs of developed LV failure, but not RV failure, corresponding to the transition stage from the compensated to the decompensated HF. Each of the treatment regimens attenuated the increase in LV end-diastolic volume, LV end-diastolic pressure, and LV relaxation constant tau (a measure of preload-independent isovolumic relaxation). On the other hand, the systolic and diastolic function parameters, including total power output and LV wall stress, were not favorably changed and did not come close to the values observed in sham-operated TGR. Moreover, the treatment with ACEi, alone or combined with EET-A, significantly attenuated lung congestion in ACF TGR. In contrast, after EET-A alone, the changes did not reach the statistical significance level. However, it is noteworthy that the combined treatment with EET-A and ACEi tended to reduce lung congestion, especially in ACF TGR groups. Taken together, our present findings indicate that all the treatment regimens exhibited some favorable actions on the diastolic function of the LV and attenuated the development of lung congestion in ACF TGR. This improvement was likely responsible for the reduction of HF-related mortality. This would be in agreement with our recent findings that in ACF TGR renal denervation markedly attenuated bilateral cardiac hypertrophy and lung congestion, leading to the conclusion that RDN might be responsible for the improvement of long-term survival [31]. Nevertheless, it is important to acknowledge that our data obtained by echocardiographic analysis in animals that survived until the end of the study after comparing to the results obtained in the very early phase of HF (see Tables 2 and 4) clearly show that treatment with ACEi, alone or combined with EET-A did not prevent the progression of development of dilated cardiomyopathy, which is one of the hallmarks in this model of high-out HF $[28,29,33,34]$.

Our present and previous results give rise to a crucial question regarding the actual mechanism(s) responsible for the beneficial effects of the treatments applied on LV enddiastolic pressure and lung congestion. Such favorable results were also present at the end of the experiment in the ACF TGR treated with the combination of EET-A and ACEi. Remarkably, at this critical stage, the treatment did not further reduce TPR. Nor did it further decrease TPR at the end of the study compared with groups treated with EET-A or ACEi alone. Therefore, the observed beneficial actions cannot be simply ascribed to reduced afterload. This line of reasoning accords well with our recent findings showing that RDN in ACF TGR exhibited similar beneficial effects but did not alter MAP [31]. Admittedly, we cannot draw decisive conclusions regarding the mechanism(s) responsible for the beneficial actions of the treatments on the LV end-diastolic pressure, LV end-diastolic volume, LV isovolumic relaxation, and, consequently, on lung congestion in the critical phase of HF (transition from compensation to the decompensation phase) in the ACF TGR; further studies are needed to elucidate this issue. 


\section{Conclusions}

Despite the apparent limitations of this study, our data show that the ACF TGR in the early phase of high-output HF exhibits substantial tissue deficiency of EETs. This might importantly contribute to developing "cardiorenal syndrome" and progression of HF in this model to the fatal end. There is no doubt that EET-A treatment delays the onset of decompensation of HF and improves the survival rate in ACF TGR; hence, we believe that it could be a promising novel therapeutic approach for the treatment of HF. Admittedly, after the addition of EET-A to ACEi treatment, the survival of ACF TGR only tended to improve compared with the effects of EET-A or ACEi given alone.

Our findings indicate that although ACF TGR develops severe renal dysfunction, the protective effects of either EET-A or ACEi treatments are not dominantly achieved by renal mechanism(s). More likely, beneficial actions on long-term survival are mediated by improving cardiac function, i.e., by reducing bilateral cardiac hypertrophy and lung congestion. This was particularly pronounced after the combined treatment with EET$\mathrm{A}$ and ACEi; however, the specific underlying mechanism(s) remain to be elucidated. Whatever the exact mechanism(s), we believe that our present data strongly support the notion that targeting the CYP-dependent epoxygenase pathway of AA should be considered in attempts to develop new pharmacological strategies for HF treatment.

Supplementary Materials: The following are available online at https:/ / www.mdpi.com/article/10 $.3390 /$ biomedicines $9081053 /$ s1.

Author Contributions: All authors conceived and designed the study. P.K. and M.M. participated in all experiments in each series; Š.J., Z.V., H.M., O.G., E.K.-J. performed the mRNA expression, biochemical, and Western blot studies; J.D.I. and J.R.F. designed, developed, and synthesized; EET-A. P.Š. and P.K. participated in the renal function studies; P.K., R.A., V.G., M.V. and M.T. performed analyses of cardiac function, echocardiography and pressure-volume analyses; P.K., J.S., J.D.I., J.V., L.C.. and M.T. were responsible for interpreting the obtained results with potential implications to the clinical practice. All authors have read and agreed to the published version of the manuscript.

Funding: This study was primarily supported by the Ministry of Health of the Czech Republic grant No. 17-28220A awarded to M. Táborský. Petr Kala is a Ph.D. student supported by the Grant Agency of Charles University, project number 68121. John Falck was supported by the 27 Robert A. Welch Foundation (I-0011). A Dr. Ralph and Marian Falk Medical Research Trust Bank of America, N.A., Trustee Grant to John D. Imig and John R. Falck provided support.

Institutional Review Board Statement: The studies followed the guidelines and practices established by the Animal Care and Use Committee of the IKEM, which accord with the national law and with American Physiological Society guiding principles for the care and use of vertebrate animals in research and training, and were approved by the Animal Care and Use Committee of the IKEM (March 2016) and, consequently, by the Ministry of Health of the Czech Republic (project decision $36388 / 2019-4 /)$.

Informed Consent Statement: Not applicable.

Data Availability Statement: Data is contained within the article and supplementary material (Supplemtary Figures S1-S6).

Conflicts of Interest: John D. Imig and John R. Falck have patents that cover the composition of matter for EET-A. There are no other conflicts of interest, financial or otherwise, are declared by the authors. 


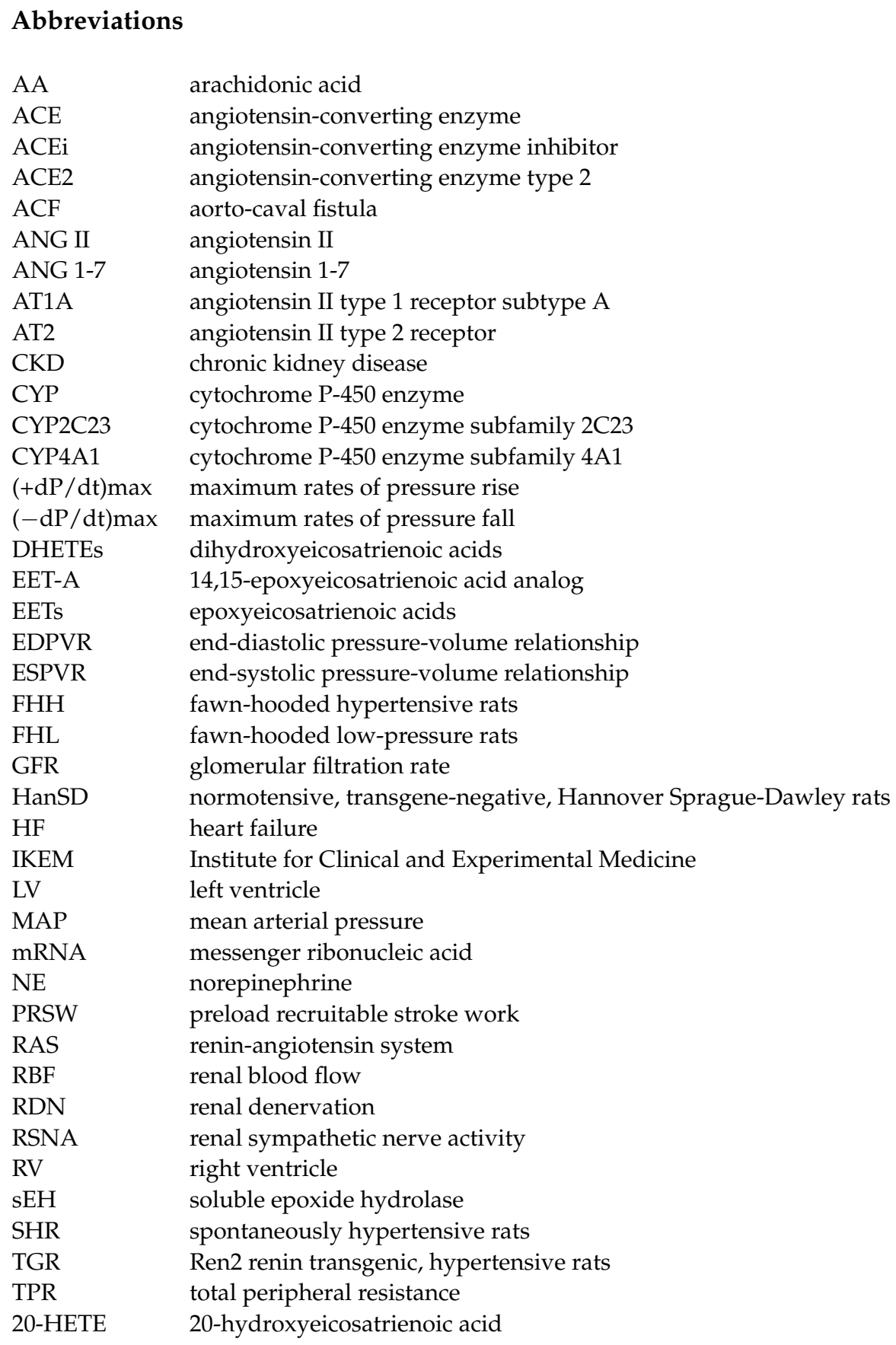

\section{References}

1. Ponikowski, P.; Voors, A.A.; Anker, S.D.; Bueno, H.; Cleland, J.G.; Coats, A.J.; Falk, V.; González-Juanaatey, J.R.; Harjola, V.-P.; Jankowska, E.A.; et al. 2016 ESC Guidelines for the diagnosis and treatment of acute and chronic heart failure: The Task Force for the diagnosis and treatment of acute and chronic heart failure of the European Society of Cardiology (ESC). Developed with the special contribution of the Heart Failure Association (HFA) of the ESC. Eur. Heart J. 2016, 37, 2129-2200.

2. Benjamin, E.J.; Blaha, M.J.; Chiuve, S.E.; Cushman, M.; Das, S.R.; Deo, R.; de Ferranti, S.D.; Floyd, J.; Fornage, M.; Gillespie, C.; et al. Heart disease and stroke statistics-2017 update: A report from the American heart association. Circulation 2017, 135, e146-e603. [CrossRef]

3. Hein, A.; Scialla, J.; Edmonston, D.; Cooper, L.B.; DeVore, A.D.; Mentz, R.J. Medical management of heart failure with reduced ejection fraction in patients with advanced renal disease. JACC Heart Fail. 2019, 7, 371-382. [CrossRef]

4. Vaduganathan, M.; Claggett, B.L.; Jhund, P.S.; Cunningham, J.W.; Ferreira, J.P.; Zannad, F.; Packer, M.; Fonarow, G.C.; McMurray, J.J.V.; Solomon, S.D. Estimating lifetime benefits of comprehensive disease-modifying pharmacological therapies in patients with heart failure with reduced ejection fraction: A comparative analysis of three randomised controlled trials. Lancet 2020, 396, 121-128. [CrossRef] 
5. Packer, M.; Anker, S.D.; Butler, J.; Filippatos, G.; Pocock, S.J.; Carson, P.; Januzzi, J.; Verma, S.; Tsutsui, H.; Brueckman, M.; et al. EMPEROR reduced trial investigators cardiovascular and renal outcomes with empagli-flozin in heart failure. N. Eng. J. Med. 2020, 383, 1413-1424. [CrossRef] [PubMed]

6. Murphy, S.P.; Ibrahim, N.E.; Januzzi, J.J. Heart failure with reduced ejection fraction. JAMA 2020, 324, 488-504. [CrossRef] [PubMed]

7. Imig, J.D. Epoxyeicosanoids in Hypertension. Physiol. Res. 2019, 68, 695-704. [CrossRef] [PubMed]

8. Elmarakby, A.A. Reno-protective mechanisms of epoxyeicosatrienoic acids in cardiovascular disease. Am. J. Physiol. Integr. Comp. Physiol. 2012, 302, R321-R330. [CrossRef]

9. Fan, F.; Roman, R.J. Effect of cytochrome P450 metabolites of arachidonic acid in nephrology. J. Am. Soc. Nephrol. 2017, 28, 2845-2855. [CrossRef] [PubMed]

10. Jamieson, K.L.; Endo, T.; Darwesh, A.M.; Samokhvalov, V.; Seubert, J.M. Cytochrome P450-derived eicosanoids and heart function. Pharmacol. Ther. 2017, 179, 47-83. [CrossRef] [PubMed]

11. Sporková, A.; Reddy, R.M.; Falck, J.R.; Imig, J.D.; Kopkan, L.; Sadowski, J.; Červenka, L. Interlobular arteries from 2-kidney, 1-clip Goldblatt hypertensive ras' exhibit-impaired vasodilatory response to epoxyeicosatrienoic acids. Am. J. Med. Sci. 2016, 351, 513-519. [CrossRef] [PubMed]

12. Rangawwami, J.; Bhalla, V.; Blair, J.E.A.; Chang, T.I.; Costa, S.; Lentine, K.L.; Lerma, E.V.; Mezeu, K.; Molitch, M.; Mullens, W.; et al. Cardiorenal syndrome: Classification, pathophysiology, diagnosis, and treatment strategies. Circulation 2019, 139, e840-e878.

13. Messerli, F.H.; Rimoldi, S.F.; Bangalore, S. The transition from hypertension to heart failure. JACC Heart Fail. $2017,5,543-551$. [CrossRef]

14. Dube, P.; Weber, K.T. Congestive heart failure: Pathophysiologic consequences of neurohormonal activation and the potential for recovery: Part I. Am. J. Med. Sci. 2011, 342, 348-351. [CrossRef]

15. Packer, M. The neurohormonal hypothesis: A theory to explain the mechanism of disease progression in heart failure. J. Am. Coll. Cardiol. 1992, 20, 248-254. [CrossRef]

16. Pavo, N.; Prausmüller, S.; Spinka, G.; Goliasch, G.; Bartko, P.E.; Wurm, R.; Arfsten, H.; Strunk, G.; Poglitsch, M.; Domenig, O.; et al. Myocardial angiotensin metabolism in end-stage heart failure. J. Am. Coll. Cardiol. 2021, 77, 1731-1743. [CrossRef]

17. Fleming, I. The pharmacology of the cytochrome P450 epoxygenase/soluble epoxide hydrolase axis in the vasculature and cardiovascular disease. Pharmacol. Rev. 2014, 66, 1106-1140. [CrossRef] [PubMed]

18. Ma, Y.H.; Schwartzman, M.L.; Roman, R.J. Altered renal P-450 metabolism of arachidonic acid in Dahl salt-sensitive rats. Am. J. Physiol. Integr. Comp. Physiol. 1994, 267, R579-R589. [CrossRef]

19. Kaergel, E.; Muller, D.N.; Honeck, H.; Theuer, J.; Shagdarsuren, E.; Mullally, A.; Luft, F.; Schunck, W.-H. P450-dependent arachidonic acid metabolism and angiotensin II-induced renal damage. Hypertens 2002, 40, 273-279. [CrossRef] [PubMed]

20. Falck, J.R.; Kodela, R.; Manne, R.; Atcha, R.; Puli, N.; Dubasi, N. 14,15-Epoxyeicosa-5,8,11-trienoic acid (14,15-EET) surro-gates containing epoxide bioisosteres: Influence upon vascular relaxation and soluble epoxide hydrolase inhibition. J. Med. Chem. 2009, 52, 5069-5075. [CrossRef] [PubMed]

21. Imig, J.D.; Elmarakby, A.; Nithipatikom, K.; Wei, S.; Capdevila, J.H.; Tuniki, V.R.; Sangras, B.; Anjaiah, S.; Manthati, V.L.; Reddy, D.S.; et al. Development of epoxyeicosatrienoic acid analogs with in vivo anti-hypertensive actions. Front. Physiol. $2010,1,157$. [CrossRef]

22. Hrdlička, J.; Neckář, J.; Papoušek, F.; Husková, Z.; Kikerlová, S.; Vaňourková, Z.; Vernerová, Z.; Akat, F.; Vašinová, J.; Hammock, B.D.; et al. Epoxyeicosatrienoic acid-based therapy attenuates the progression of postischemic heart failure in normotensive sprague-dawley but not in hypertensive Ren-2 transgenic Rats. Front. Pharmacol. 2019, 10, 159. [CrossRef]

23. Neckář, J.; Khan, M.A.H.; Gross, G.J.; Cyprová, M.; Hrdlička, J.; Kvasilová, A.; Falck, J.R.; Campbell, W.B.; Sedláková, L.; Škutová, S.; et al. Epoxyeicosatrienoic acid analog EET-B at-tenuates post-myocardial infarction remodeling in spontaneously hypertensive rats. Clin. Sci. 2019, 133, 939-951. [CrossRef]

24. Imig, J.D.; Khan, M.A.H.; Burkhan, A.; Chen, G.; Adebesin, A.M.; Falck, J.R. Kidney-targeting epoxyeicosatrienoic acid ana-log, EET-F01, reduces inflammation, oxidative stress, and cisplatin-induced nephrotoxicity. Int. J. Mol. Sci. 2021, 22, 2793. [CrossRef] [PubMed]

25. Walkowska, A.; Červenka, L.; Imig, J.D.; Falck, J.R.; Sadowski, J.; Kompanowska-Jezierska, E. Early Renal Vasodilator and Hypotensive Action of Epoxyeicosatrienoic Acid Analog (EET-A) and 20-HETE Receptor Blocker (AAA) in Spontaneously Hypertensive Rats. Front. Physiol. 2021, 12. [CrossRef]

26. Garcia, R.; Diebold, S. Simple, rapid, and effective method of producing aorto-caval shunts in the rat. Cardiovasc. Res. 1990, 24, 430-432. [CrossRef] [PubMed]

27. Cohen-Segev, R.; Francis, B.; Abu-Saleh, N.; Awad, H.; Lazarovich, A.; Kabala, A.; Aronson, D.; Abassi, Z. Cardiac and renal distribution of ACE and ACE-2 in rats with heart failure. Acta Histochem. 2014, 116, 1342-1349. [CrossRef] [PubMed]

28. Abassi, Z.; Goltsman, I.; Karram, T.; Winaver, J.; Hoffman, A. Aortocaval Fistula in Rat: A Unique Model of Volume-Overload Congestive Heart Failure and Cardiac Hypertrophy. J. Biomed. Biotechnol. 2011, 2011, 729497. [CrossRef]

29. Brower, G.; Levick, S.; Janicki, J.S. Differential Effects of Prevention and Reversal Treatment with Lisinopril on Left Ventricular Remodelling in a Rat Model of Heart Failure. Hear. Lung Circ. 2015, 24, 919-924. [CrossRef] 
30. Kala, P.; Sedláková, L.; Škaroupková, P.; Kopkan, L.; Vaňourková, Z.; Táborský, M.; Nishiyama, A.; Hwang, S.H.; Hammock, B.D.; Sadowski, J.; et al. Effect of Angiotensin-Converting Enzyme Blockade, Alone or Combined With Blockade of Soluble Epoxide Hydrolase, on the Course of Congestive Heart Failure and Occurrence of Renal Dysfunction in Ren-2 Transgenic Hypertensive Rats With Aorto-Caval Fistula. Physiol. Res. 2017, 67, 401-415. [CrossRef]

31. Honetschlagerová, Z.; Gawrys, O.; Jíchová, Š.; Škaroupková, P.; Kikerlová, S.; Vaňourková, Z.; Husková, Z.; Melenovský, V.; Kom-panowska-Jezierska, E.; Sadowski, J.; et al. Renal sympathetic denervation at-tenuates congestive heart failure in angiotensin II-dependent hypertension: Studies with Ren-2 transgenic hypertensive rats with aorto-caval fistula. Kidney Blood Press. Res. 2021, 46, 95-113. [PubMed]

32. Honetschlagerová, Z.; Škaroupková, P.; Kikerlová, S.; Husková, Z.; Maxová, H.; Melenovský, V.; Kompanowska-Jezierska, E.; Sadowski, J.; Gawrys, O.; Kujal, P.; et al. Effects of renal sympathetic denervation on the course of congestive heart failure combined with chronic kidney disease: Insight from studies with fawn-hooded hypertensive rats with volume overload induced using aorto-caval fistula. Clin. Exp. Hypertens. 2021, 43, 1-14. [CrossRef] [PubMed]

33. Houser, S.R.; Margulies, K.B.; Murphy, A.M.; Spinale, F.G.; Francis, G.S.; Prabhu, S.D. Animal models of heart failure: A sci-entific statement from the American Heart Association. Circ. Res. 2012, 111, 131-150. [CrossRef] [PubMed]

34. Riehle, C.; Bauersachs, J. Small animal models of heart failure. Cardiovasc. Res. 2019, 115, 1838-1849. [CrossRef] [PubMed]

35. Mullins, J.J.; Peters, J.F.; Ganten, D. Fulminant hypertension in transgenic rats harbouring the mouse Ren-2 gene. Nat. Cell Biol. 1990, 344, 541-544. [CrossRef] [PubMed]

36. Husková, Z.; Kramer, H.J.; Vaňourková, Z.; Červenka, L. Effects of changes in sodium balance on plasma and kidney angio-tensin II levels in anesthetized and conscious Ren-2 transgenic rats. J. Hypertens. 2006, 24, 517-527. [CrossRef]

37. Červenka, L.; Melenovský, V.; Husková, Z.; Škaroupková, P.; Nishiyama, A.; Sadowski, J. Inhibition of soluble epoxide hydrolase counteracts the development of renal dysfunction and progression of congestive heart failure in Ren-2 transgenic hyperten-sive rats with aorto-caval fistula. Clin. Exp. Pharmacol. Physiol. 2015, 42, 795-807. [CrossRef]

38. Jíchová, Š.; Kopkan, L.; Husková, Z.; Doleželová, Š.; Neckář, J.; Kujal, P.; Verenerová, Z.; Kramer, H.J.; Sadowski, J.; Kompanowska-Jezierska, E.; et al. Epoxyeicosatrienoic acid analog attenuates the develop-ment of malignant hypertension, but does not reverse it once established: A study in Cyp1a1-Ren-2 transgenic rats. J. Hypertens. 2016, 34, 2008-2025. [CrossRef]

39. Červenka, L.; Husková, Z.; Kopkan, L.; Kikerlová, S.; Sedláková, L.; Vaňourková, Z.; Alánová, P.; Kolář, F.; Hammock, B.D.; Hwang, S.H.; et al. Two pharmacological epoxyeicosatrienoic ac-id-enhancing therapies are effectively antihypertensive and reduce the severity of ischemic arrhythmias in rats with angio-tensin II-dependent hypertension. J. Hypertens. 2018, 3, $1326-1341$. [CrossRef]

40. Gawrys, O.; Husková, Z.; Baranowska, I.; Walkowska, A.; Sadowski Kikerlová, S.; Vaňourková, Z.; Honetschlagerová, Z.; Ška-roupková, P.; Červenka, L.; Falck, J.; et al. Combined treatment with epoxyeicosatrienoic acid an-alog and 20hydroxyeicosatetraenoic acid antagonist provides substantial hypotensive effect in spontaneously hypertensive rats. J. Hypertens. 2020, 38, 1802-1810. [CrossRef]

41. Kala, P.; Červenka, L.; Škaroupková, P.; Táborský, M.; Kompanowska-Jezierska, E.; Sadowski, J. Sex-linked differences in the mor-tality in Ren-2 transgenic hypertensive rats with aorto-caval fistula: Effects of treatment with angiotensin-converting enzyme alone and combined with inhibitor of soluble epoxide hydrolase. Physiol. Res. 2019, 68, 589-601. [CrossRef] [PubMed]

42. Hillege, H.L.; Girbes, A.R.; de Kam, P.J.; Boomsma, F.; de Zeeuw, D.; Charlesworth, A.; Hampton, J.R.; van Veldhuisen, D.J. Renal func-tion, neurohormonal activation, and survival in patients with chronic heart failure. Circulation 2000, 102, 203-210. [CrossRef]

43. Schefold, J.C.; Filippatos, G.; Hasenfuss, G.; Anker, S.D.; von Haehling, S. Heart failure and kidney dysfunction: Epidemiolo-gy, mechanisms and management. Nat. Rev. Cardiol. 2016, 12, 610-623. [CrossRef] [PubMed]

44. Khayyat-Kholghi, M.; Oparil, S.; Davis, B.R.; Tereshchenko, L.G. Worsening kidney function is the major mechanism of heart fail-ure in hypertension. The ALLHAT study. JACC Heart Fail. 2021, 9, 100-111. [CrossRef] [PubMed]

45. Antoine, S.; Vaidya, G.; Imam, H.; Villarreal, D. Pathophysiologic Mechanisms in Heart Failure: Role of the Sympathetic Nervous System. Am. J. Med. Sci. 2017, 353, 27-30. [CrossRef] [PubMed]

46. Sharp, T.E., III; Lefer, D.J. Renal denervation to treat heart failure. Annu. Rev. Physiol. 2021, 83, 4.1-4.20. [CrossRef]

47. Roman, R.J.; Fan, F. 20-HETE. Hypertension and beyond. Hypertension 2018, 72, 12-18. [CrossRef] [PubMed]

48. Alsaad, A.M.S.; Zordoky, B.; Tse, M.M.Y.; El-Kadi, A.O.S. Role of cytochrome P450-mediated arachidonic acid metabolites in the pathogenesis of cardiac hypertrophy. Drug Metab. Rev. 2013, 45, 173-195. [CrossRef] [PubMed]

49. Rocic, P.; Schwartzman, M.L. 20-HETE in the regulation of vascular and cardiac function. Pharmacol. Ther. 2018, 192, 74-87. [CrossRef]

50. El-Sherbeni, A.A.; Aboutabl, M.E.; Zordoky, B.N.M.; Anwa-Mohamed, A.; El-Kadi, A.O.S. Determination of the dominant arachidonic acid cytochrome P450 monooxygenase in rat heart, lung, kidney and liver: Protein expression and metabolic kinetics. AAPS J. 2013, 15, 112-122. [CrossRef]

51. Kratky, V.; Vanourkova, Z.; Sykora, M.; Bacova, B.S.; Hruskova, Z.; Kikerlova, S.; Huskova, Z.; Kopkan, L. AT1 receptor blocker, but not an ACE inhibitor, prevents kidneys from hypoperfusion during congestive heart failure in normotensive and hypertensive rats. Sci. Rep. 2021, 11, 1-15. [CrossRef] [PubMed]

52. Livak, K.J.; Schmittgen, T.D. Analysis of relative gene expression data using real-time quanti-tative PCR and the 2(-Delta Delta C(T)) Method. Methods 2001, 41, 402-408. [CrossRef] [PubMed] 
53. Bas, A.; Forsberg, G.; Hammarstrom, S.; Hammarstrom, M.L. Utility of the housekeeping genes 18S rRNA, beta-actin and glycer-aldehyde-3-phosphate-dehydrogenase for normalization in real-time quantitative reverse transcriptase-polymerase chain re-action analysis of gene expression in human T lymphocytes. Scand. J. Immunol. 2004, 59, 566-573. [CrossRef] [PubMed]

54. Jíchová, Š.; Doleželová, Š.; Kopkan, L.; Kompanowska-Jezierska, E.; Sadowski, J.; Červenka, L. Fenofibrate Attenuates Malignant Hypertension by Suppression of the Renin-angiotensin System: A Study in Cyp1a1-Ren-2 Transgenic Rats. Am. J. Med. Sci. 2016, 352, 618-630. [CrossRef] [PubMed]

55. Husková, Z.; Kramer, H.J.; Thumová, M.; Vaňourková, Z.; Bürgelová, M.; Teplan, V.; Malý, J.; Červenka, L. Effects of anaesthesia on plasma and kidney ANG II levels in normotensive and ANG II-dependent hypertensive rats. Kidney Blood Res. 2006, 29 , 74-83. [CrossRef] [PubMed]

56. Husková, Z.; Kopkan, L.; Červenková, L.; Doleželová, Š.; Vaňourková, Z.; Škaroupková, P.; Nishiyama, A.; KompanowskaJezierska, E.; Sadowski, J.; Kramer, H.J.; et al. Intrarenal alterations of the angiotensin-converting enzyme type 2/angiotensin 1-7 complex of the renin-angiotensin system do not alter the course of malignant hypertension in Cyp1a1-Ren-2 transgenic rats. Clin. Exp. Pharmacol. Physiol. 2016, 43, 438-449. [CrossRef]

57. Kala, P.; Bartušková, H.; Pit’ha, J.; Vaňourková, Z.; Kikerlová, S.; Jíchová, Š.; Melenovský, V.; Hošková, L.; Veselka, J.; Kompanowska-Jezierska, E.; et al. Deleterious effects of hyperactivity of the ren-in-angiotensin system and hypertension on the course of chemotherapy-induced heart failure after doxorubicin administra-tion: A study in Ren-2 transgenic rats. Int. J. Mol. Sci. 2020, 2, 9337. [CrossRef]

58. Cohen, J. (Ed.) Some issue in power analysis. In Statistical Power Analysis for Bevavioral Sciences, 2nd ed.; Routledge: Oxford, UK, 2013; pp. 531-542.

59. Červenka, L.; Melenovský, V.; Husková, Z.; Sporková, A.; Burgelová, M.; Škaroupková, P.; Hwang, S.H.; Hammock, B.D.; Imig, J.D.; Sadowski, J. Inhibition of soluble epoxide hydrolase does not improve the course of congestive heart failure and the develop-ment of renal dysfunction in rats with volume overload induced by aorto-caval fistula. Physiol. Res. 2015, 64, 857-873. [CrossRef]

60. Kratky, V.; Kopkan, L.; Kikerlova, S.; Huskova, Z.; Taborsky, M.; Sadowski, J.; Kolar, F.; Cervenka, L. The role of renal vascular reac-tivity in the development of renal dysfunction in compensated and decompensated congestive heart failure. Kidney Blood Press. Res. 2018, 43, 1730-1741. [CrossRef]

61. Yin, F.C.P.; Spurgeon, H.A.; Rakusan, K.; Weisfeldt, M.L.; Lakatta, E.G. Use of tibia length to quantify cardiac hypertrophy: Application in the aging rat. Am. J. Physiol. 1982, 243, H941-H947.

62. Vaňourková, Z.; Kramer, H.J.; Husková, Z.; Vaněčková, I.; Opočenský, M.; Čertíková Chábová, V.; Tesař, V.; Škaroupková, P.; Thu-mová, M.; Dohnalová, M.; et al. AT1 receptor blockade is superiod to convetional triple therapy in protecting against end-organ damage in Cyp1a1-Ren-2 transgenic rats with inducible hypertension. J. Hypertens. 2006, 24, 2465-2472. [CrossRef]

63. Hartupee, J.; Mann, D.L. Neurohormonal activation in heart failure with reduced ejection fraction. Nat. Rev. Cardiol. 2017, 14, 30-38. [CrossRef]

64. Packer, M.; McMurray, J.J. Importance of endogenous compensatory vasoactive peptides in broadening the effects of inhibi-tors of the renin-angiotensin system for the treatment of heart failure. Lancet 2017, 389, 1831-1840. [CrossRef]

65. Díaz, H.S.; Toledo, C.; Andrade, D.C.; Marcus, N.J.; Del Rio, R. Neuroinflammation in heart failure: New insights for an old disease. J. Physiol. 2019, 598, 33-59. [CrossRef]

66. Wang, K.; Basu, R.; Poglitsch, M.; Bakal, J.A.; Stat, P.; Oudit, G.Y. Elevated angiotensin 1-7/angiotensin II ratio predicts fa-vorable outcomes in patients with heart failure. Circ. Heart Fail. 2020, 13, e006939. [CrossRef] [PubMed]

67. Vacková, Š.; Kikerlová, S.; Melenovsky, V.; Kolar, F.; Imig, J.; Kompanowska-Jezierska, E.; Sadowski, J.; Červenka, L. Altered Renal Vascular Responsiveness to Vasoactive Agents in Rats with Angiotensin II-Dependent Hypertension and Congestive Heart Failure. Kidney Blood Press. Res. 2019, 44, 792-809. [CrossRef]

68. Melenovsky, V.; Skaroupkova, P.; Benes, J.; Torresova, V.; Kopkan, L.; Cervenka, L. The Course of Heart Failure Development and Mortality in Rats with Volume Overload due to Aorto-Caval Fistula. Kidney Blood Press. Res. 2012, 35, 167-173. [CrossRef] [PubMed]

69. Oka, T.; Nishimura, H.; Ueyama, M.; Kubota, J.; Kawamura, K. Haemodynamic and neurohormonal changes in spontane-ously hypertensive rats with aorto-caval fistula. Clin. Sci. 1993, 84, 531-535. [CrossRef]

70. Lee, M.A.; Bohm, M.; Paul, M.; Bader, M.; Ganten, U.; Ganten, D. Physiological characterization of the hypertensive trans-genic rat TGR(mREN2)27. Am. J. Physiol. 1996, 270, E919-E929.

71. Langheinrich, M.; Lee, M.A.; Bohm, M.; Pinto, Y.M.; Ganten, D.; Paul, M. Hypertensive Ren-2 transgenic rat TGR(mREN2)27 in hypertension research. Characteristic and functional aspects. Am. J. Hypertens. 1996, 9, 506-512. [CrossRef]

72. Lush, D.J.; King, J.A.; Fray, J.C. Pathophysiology of low renin syndromes: Sites of renal renin secretory impairment and prorenin overexpression. Kidney Int. 1993, 43, 983-999. [CrossRef] [PubMed]

73. Lai, J.; Chen, C. The Role of Epoxyeicosatrienoic Acids in Cardiac Remodeling. Front. Physiol. 2021, 12, 642470. [CrossRef]

74. Kim, G.; Uriel, N.; Burkhoff, D. Reverse remodelling and myocardial recovery in heart failure. Nat. Rev. Cardiol. 2018, 15, 83-96. [CrossRef] [PubMed] 
75. Sporková, A.; Husková, Z.; Škaroupková, P.; Reddy, R.N.; Falck, J.R.; Sadowski, J.; Červenka, L. Vasodilatory responses of renal interlobular arteries to epoxyeicosatrienoic acids analog are not enhanced in Ren-2 transgenic hypertensive rats: Evi-dence against a role of direct vascular effects of epoxyeicosatrienoic acids in the progression of experimental heart failure. Physiol. Res. 2017, 66, 29-39. [PubMed]

76. Ruzicka, M.; Yuan, B.; Harmsen, E.; Leenen, F.H. The renin-angiotensin system and volume overload-induced cardiac hypertrophy in rats. Effects of angiotensin converting enzyme inhibitor versus angiotensin II receptor blocker. Circulation 1993, 87, 921-930. [CrossRef]

77. Ruzicka, M.; Yuan, B.; Leenen, F.H.H. Effects of enalapril versus losartan on regression of volume overload-induced cardiac hypertrophy in rats. Circulation 1994, 90, 484-491. [CrossRef] 